\title{
EXPRESSIVE LOGICS FOR COINDUCTIVE PREDICATES
}

\author{
CLEMENS KUPKE $^{a}$ AND JURRIAAN ROT ${ }^{b}$ \\ ${ }^{a}$ Department of Computer \& Information Sciences, Strathclyde University, United Kingdom \\ e-mail address: clemens.kupke@strath.ac.uk \\ ${ }^{b}$ Institute for Computing and Information Sciences (iCIS), Radboud University, The Netherlands \\ e-mail address: jrot@cs.ru.nl
}

\begin{abstract}
The classical Hennessy-Milner theorem says that two states of an image-finite transition system are bisimilar if and only if they satisfy the same formulas in a certain modal logic. In this paper we study this type of result in a general context, moving from transition systems to coalgebras and from bisimilarity to coinductive predicates. We formulate when a logic fully characterises a coinductive predicate on coalgebras, by providing suitable notions of adequacy and expressiveness, and give sufficient conditions on the semantics. The approach is illustrated with logics characterising similarity, divergence and a behavioural metric on automata.
\end{abstract}

\section{INTRODUCTION}

The deep connection between bisimilarity and modal logic manifests itself in the HennessyMilner theorem: two states of an image-finite labelled transition system (LTS) are behaviourally equivalent iff they satisfy the same formulas in a certain modal logic [HM85]. From left to right, this equivalence is sometimes referred to as adequacy of the logic w.r.t. bisimilarity, and from right to left as expressiveness. By stating both adequacy and expressiveness, the Hennessy-Milner theorem thus gives a logical characterisation of behavioural equivalence.

There are numerous variants and generalisations of this kind of result. For instance, a state $x$ of an LTS is simulated by a state $y$ if every formula satisfied by $x$ is also satisfied by $y$, where the logic only has conjunction and diamond modalities; see [vG90] for this and many other related results. Another class of examples is logical characterisations of quantitative notions of equivalence, such as probabilistic bisimilarity and behavioural distances (e.g., [LS91, DGJP99, DEP02, vBW05, JS09, KM18, WSPK18, CFKP19]). In many such cases, including bisimilarity, the comparison between states is coinductive, and the problem is thus to characterise a coinductively defined relation (or distance) with a suitable modal logic.

Key words and phrases: Coalgebra, Fibration, Modal Logic.

* This is a revised and extended version of a paper which appeared in the proceedings of CSL 2020 [KR20]. Part of this research was carried out during the second author's stay at University College London, funded by the European Union's Horizon 2020 research and innovation programme under the Marie Skłodowska-Curie Grant Agreement No. 795119. 
Both coinduction and modal logic can be naturally and generally studied within the theory of coalgebra, which provides an abstract, uniform study of state-based systems [Rut00, Jac16]. Indeed, in the area of coalgebraic modal logic [KP11] there is a rich literature on deriving expressive logics for behavioural equivalence between state-based systems, thus going well beyond labelled transition systems [Pat04, Sch08, Kli07]. However, such results focus almost exclusively on behavioural equivalence or bisimilarity - a coalgebraic theory of logics for characterising coinductive predicates other than bisimilarity is still missing. The aim of this paper is to accommodate the study of logical characterisation of coinductive predicates in a general manner, and provide tools to prove adequacy and expressiveness.

Our approach is based on universal coalgebra, to achieve results that apply generally to state-based systems. Central to the approach are the following two ingredients.

(1) Coinductive predicates in a fibration. To characterise coinductive predicates, we make use of fibrations - this approach originates from the seminal work of Hermida and Jacobs [HJ98]. The fibration is used to speak about predicates and relations on states. In this context, liftings of the type functor of coalgebras uniformly determine coinductive predicates and relations on such coalgebras. An important feature of this approach, advocated in [HKC18], is that it covers not only bisimilarity, but also other coinductive predicates including, e.g., similarity of labelled transition systems and other coalgebras [HJ04], behavioural metrics [BBKK18, BKP18, SKDH18], unary predicates such as divergence [BPPR17, HKC18], and many more.

(2) Coalgebraic modal logic via dual adjunctions. We use an abstract formulation of coalgebraic logic, which originated in [PMW06, Kli07], building on a tradition of logics via duality (e.g., [KKP04, BK05a]). This framework is formulated in terms of a contravariant adjunction, which captures the basic connection between states and theories, and a distributive law, which captures the one-step semantics of the logic. It covers classical modal logics of course, but also easily accommodates multi-valued logics, and, e.g., logics without propositional connectives, where formulas can be thought of as basic tests on state-based systems. This makes the framework suitable for an abstract formulation of Hennessy-Milner type theorems, where formulas play the role of tests on state-based systems.

To formulate adequacy and expressiveness with respect to general coinductive predicates, we need to know how to compare collections of formulas. For instance, if the coinductive predicate is similarity of LTSs, then the associated logical theories of one state should be included in the other, not necessarily equal. This amounts to stipulating a relation on truth values, that extends to a relation between theories. In the quantitative case, we need a logical distance between collections of formulas; this typically arises from a distance between truth values (which, in this case, will typically be an interval in the real numbers). The fibrational setting provides a convenient means for defining such an object for comparing theories.

With this in hand, we arrive at the main contributions of this paper: the formulation of adequacy and expressiveness of a coalgebraic modal logic with respect to a coinductive predicate in a fibration, and sufficient conditions on the semantics of the logic that guarantee adequacy and expressiveness. We exemplify the approach through a range of examples, including logical characterisations of a simple behavioural distance on deterministic automata, similarity of labelled transition systems, and a logical characterisation of a unary predicate: divergence, the set of states of an LTS which have an infinite path of outgoing $\tau$-steps. The 
latter is characterised, on image-finite LTSs, by a quantitative logic with only diamond formulas, i.e., the set of formulas is simply the set of words.

Related work. As mentioned above, there are numerous specific results on Hennessy-Milner theorems, which - e.g., in the probabilistic setting as in [CFKP19] - can be highly non-trivial. A comprehensive historical treatment is beyond the scope of this paper, which is, instead, broad: it aims at studying these kinds of results in a general, coalgebraic setting.

The case of capturing bisimilarity and behavioural equivalence of coalgebras by modal logics has been very well studied, see [KP11] for an overview. Expressiveness w.r.t. similarity has been studied in [KKV12], which is close in spirit to our approach, but focuses on the poset case. On a detailed level, the logic for similarity is based on distributive lattices, hence it uses disjunction; this differs from our example, which only uses conjunction and diamond modalities. Another study of expressiveness of logics w.r.t. various forms of similarity is in [Wil13]. Expressiveness of multi-valued coalgebraic logics w.r.t. behavioural equivalence is studied in [BD16]. In [BH17], notions of equivalence are extracted from a logic through a variant of $\Lambda$-bisimulation [GS13]. To the best of our knowledge, the current work is the first in the area that connects general coinductive predicates in a fibration to coalgebraic logics.

In the recent [DMS19], the authors prove Hennessy-Milner type theorems for coalgebras including, but going significantly beyond bisimilarity. The logics are related to a semantics obtained from graded monads. The scope differs substantially from the current paper: the graded monad approach is inductive and focuses on semantic equivalence of different types, whereas our framework aims at characterising coinductive predicates, and in our approach it is essential to be able to relate theories in different ways than equivalence (to cover, e.g., similarity, divergence or logical distance). On the one hand, it appears that none of our examples can be covered immediately in loc. cit.; on the other hand, trace equivalence of various kinds can be covered in [DMS19] but not (directly) in the current paper.

In [WSPK18] a characterisation theorem is shown for fuzzy modal logic, and in [KM18] for a wide class of behavioural metrics. These papers are not aimed at other kinds of coinductive predicates, and they do not cover the examples in Section 4 (including the behavioural metric for deterministic automata, as we use a much simpler logic than in [KM18]). Conversely, the question whether the logical characterisation results of [KM18] can be covered in the current framework is left open. These papers also treat game-based characterisations of bisimilarity, which are studied in a general setting in the recent $\left[\mathrm{KKH}^{+} 19\right]$. That paper, however, does not yet feature modal logic explicitly; in fact, the connection is posed there as future work.

An earlier version of this paper appeared in the proceedings of CSL 2020 [KR20]. The current paper extends this with a more detailed treatment of the basic setup of logics in a contravariant adjunction in Section 2.3, a treatment of expressiveness of logics w.r.t. behavioural equivalence rather than bisimilarity making use of so-called lax liftings, in Section 3.2, and a new section on finite-depth expressiveness via initial and final sequences (Section 5).

Outline. The paper starts in Section 2 with preliminaries on coalgebra, fibrations and coinductive predicates, and coalgebraic modal logic. Section 3 contains the abstract framework for expressiveness and adequacy, together with sufficient conditions for establishing these. Section 4 contains three detailed examples of this setup. A different route to expressiveness, 
via initial and final sequences, is explored in Section 5. The paper concludes in Section 6 with directions for future work.

Acknowledgments. We would like to thank Fredrik Nordvall Forsberg, Ichiro Hasuo, Bart Jacobs, Shin-ya Katsumata and Yuichi Komorida for helpful discussions, comments and suggestions.

\section{Preliminaries}

The category of sets and functions is denoted by Set. The powerset functor is denoted by $\mathcal{P}$ : Set $\rightarrow$ Set, and the finite powerset functor by $\mathcal{P}_{\omega}$. The diagonal relation on a set $X$ is denoted by $\Delta_{X}=\{(x, x) \mid x \in X\}$.

Let $\mathcal{C}$ be a category, and $B: \mathcal{C} \rightarrow \mathcal{C}$ a functor. A $(B)$-coalgebra is a pair $(X, \gamma)$ where $X$ is an object in $\mathcal{C}$ and $\gamma: X \rightarrow B X$ a morphism. A homomorphism from a coalgebra $(X, \gamma)$ to a coalgebra $(Y, \theta)$ is a morphism $h: X \rightarrow Y$ such that $\theta \circ h=B h \circ \gamma$. An algebra for a functor $L: \mathcal{D} \rightarrow \mathcal{D}$ on a category $\mathcal{D}$ is a pair $(A, \alpha)$ of an object $A$ in $\mathcal{D}$ and an arrow $\alpha: L A \rightarrow A$.

Example 2.1. A labelled transition system (LTS) over a set of labels $A$ is a coalgebra $(X, \gamma)$ for the functor $B$ : Set $\rightarrow$ Set, $B X=(\mathcal{P} X)^{A}$. For states $x, x^{\prime} \in X$ and a label $a \in A$, we sometimes write $x \stackrel{a}{\rightarrow} x^{\prime}$ for $x^{\prime} \in \gamma(x)(a)$. Image-finite labelled transition systems are coalgebras for the functor $B X=\left(\mathcal{P}_{\omega} X\right)^{A}$. A deterministic automaton over an alphabet $A$ is a coalgebra for the functor $B$ : Set $\rightarrow$ Set, $B X=2 \times X^{A}$. For many other examples of state-based systems modelled as coalgebras, see, e.g., [Jac16, Rut00].

2.1. Coinductive Predicates in a Fibration. We recall the general approach to coinductive predicates in a fibration, starting by briefly presenting how bisimilarity of Set coalgebras arises in this setting (see [HKC18, HJ98, Jac16] for details). Let Rel be the category where an object is a pair $(X, R)$ consisting of a set $X$ and a relation $R \subseteq X \times X$ on it, and a morphism from $(X, R)$ to $(Y, S)$ is a map $f: X \rightarrow Y$ such that $x R y$ implies $f(x) R f(y)$, for all $x, y \in X$. Below, we sometimes refer to an object $(X, R)$ only by the relation $R \subseteq X \times X$. Any set functor $B$ : Set $\rightarrow$ Set gives rise to a functor $\operatorname{Rel}(B): \operatorname{Rel} \rightarrow \operatorname{Rel}$, defined by relation lifting:

$$
\operatorname{Rel}(B)(R \subseteq X \times X)=\left\{\left(\left(B \pi_{1}\right)(z),\left(B \pi_{2}\right)(z)\right) \in B X \times B X \mid z \in B R\right\} .
$$

Given a $B$-coalgebra $(X, \gamma)$, a bisimulation is a relation $R \subseteq X \times X$ such that $R \subseteq$ $(\gamma \times \gamma)^{-1}(\operatorname{Rel}(B)(R))$, i.e., if $x R y$ then $\gamma(x) \operatorname{Rel}(B)(R) \gamma(y)$. Bisimilarity is the greatest such relation, and equivalently, the greatest fixed point of the monotone map $R \mapsto(\gamma \times \gamma)^{-1}(\operatorname{Rel}(B)(R))$ on the complete lattice of relations on $X$, ordered by inclusion.

The functor $\operatorname{Rel}(B)$ is a lifting of $B$ : it maps a relation on $X$ to a relation on $B X$. A first step towards generalisation beyond bisimilarity is obtained by replacing $\operatorname{Rel}(B)$ by an arbitrary lifting $\bar{B}$ : Rel $\rightarrow$ Rel of $B$. For instance, for $B X=\left(\mathcal{P}_{\omega} X\right)^{A}$ one may take

$$
\bar{B}(R)=\left\{\left(t_{1}, t_{2}\right) \mid \forall a \in A . \forall x \in t_{1}(a) . \exists y \in t_{2}(a) .(x, y) \in R\right\} .
$$

Then, for an LTS $\gamma: X \rightarrow\left(\mathcal{P}_{\omega} X\right)^{A}$, the greatest fixed point of the monotone map $R \mapsto$ $(\gamma \times \gamma)^{-1} \circ \bar{B}(R)$ is similarity. In the same way, by varying the lifting $\bar{B}$, one can define many different coinductive relations on Set coalgebras. 
Yet a further generalisation is obtained by replacing Set by a general category $\mathcal{C}$, and Rel by a category of 'predicates' on $\mathcal{C}$. A suitable categorical infrastructure for such predicates on $\mathcal{C}$ is given by the notion of fibration. This allows us, for instance, to move beyond (Boolean, binary) relations to quantitative relations (e.g., behavioural metrics) or unary predicates. Such examples follow in Section 4; also see, e.g., [HKC18, BPPR17].

To define fibrations, it will be useful to fix some associated terminology first. Let $p: \mathcal{E} \rightarrow \mathcal{C}$ be a functor. If $p(R)=X$, then we say $R$ is above $X$, and similarly for morphisms. The collection of all objects $R$ above a given object $X$ and arrows above the identity id ${ }_{X}$ form a category, called the fibre above $X$ and denoted by $\mathcal{E}_{X}$.

Definition 2.2. A functor $p: \mathcal{E} \rightarrow \mathcal{C}$ is a (poset) fibration if

- each fibre $\mathcal{E}_{X}$ is a poset category (that is, at most one arrow between every two objects); the corresponding order on objects is denoted by $\leq$;

- for every $f: X \rightarrow Y$ in $\mathcal{C}$ and object $S$ above $Y$ there is a Cartesian morphism $\widetilde{f}_{S}: f^{*}(S) \rightarrow$ $S$ above $f$, with the property that for every arrow $g: Z \rightarrow X$, every object $R$ above $Z$ and arrow $h: R \rightarrow S$ above $f \circ g$, there is a unique arrow $k: R \rightarrow f^{*}(S)$ above $g$ such that $\tilde{f}_{S} \circ k=h$.

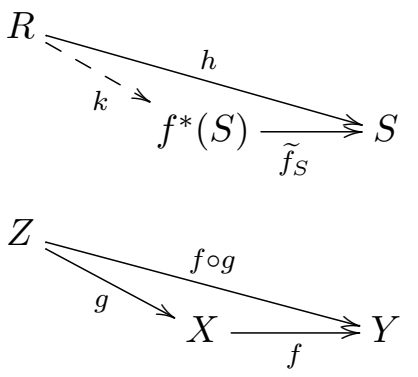

Remark 2.3. In this paper we only consider poset fibrations, and refer to them simply as fibrations. The usual definition of fibration is more general (e.g., [Jac99]): normally, fibres are not assumed to be posets. Poset fibrations have several good properties, mentioned below. In the application to coinductive predicates, it is customary to work with poset fibrations.

For a morphism $f: X \rightarrow Y$, the assignment $R \mapsto f^{*}(R)$ gives rise to a functor $f^{*}: \mathcal{E}_{Y} \rightarrow$ $\mathcal{E}_{X}$, called reindexing along $f$. (Note that functors between poset categories are just monotone maps.) We use a strengthening of poset fibrations, following [SKDH18, $\left.\mathrm{KKH}^{+} 19\right]$.

Definition 2.4. A poset fibration $p: \mathcal{E} \rightarrow \mathcal{C}$ is called a CLat $\wedge$-fibration if $\left(\mathcal{E}_{X}, \leq\right)$ is a complete lattice for every $X$, and reindexing preserves arbitrary meets.

Any poset fibration $p$ is split: we have $(g \circ f)^{*}=f^{*} \circ g^{*}$ for any morphisms $f, g$ that compose. Further, $p$ is faithful. This captures the intuition that morphisms in $\mathcal{E}$ are morphisms in $\mathcal{C}$ with a certain property; e.g., relation-preserving, or non-expansive (Examples 2.5, 2.6). We note that $\mathrm{CLat}_{\wedge}$-fibrations are instances of topological functors [Her74]. We use the former, in line with existing related work $\left[\mathrm{HKC} 18, \mathrm{KKH}^{+} 19\right]$. This also has the advantage of keeping our results amenable to possible future extensions to a wider class of examples.

Example 2.5. Consider the relation fibration $p:$ Rel $\rightarrow$ Set, where $p(R \subseteq X \times X)=X$. Reindexing is given by inverse image: for a map $f: X \rightarrow Y$ and a relation $S \subseteq Y \times Y$, we have $f^{*}(S)=(f \times f)^{-1}(S)$. The functor $p$ is a CLat $_{\wedge}$-fibration. 
Closely related is the predicate fibration $p$ : Pred $\rightarrow$ Set. An object of Pred is a pair $(X, \Gamma)$ consisting of a set $X$ and a subset $\Gamma \subseteq X$, and an arrow from $(X, \Gamma)$ to $(Y, \Theta)$ is a map $f: X \rightarrow Y$ such that $x \in \Gamma$ implies $f(x) \in \Theta$. The functor $p$ is given by $p(X, \Gamma)=X$,

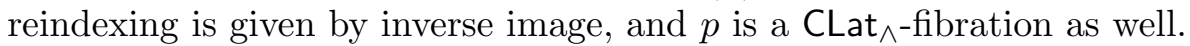

In the relation fibration, we sometimes refer to an object $\left(X, R \subseteq X^{2}\right)$ simply by $R$, and similarly in the predicate fibration.

Example 2.6. Let $\mathcal{V}$ be a complete lattice. Define the category Rel $\mathcal{V}$ as follows: an object is a pair $(X, d)$ where $X$ is a set and a function $d: X \times X \rightarrow \mathcal{V}$, and a morphism from $(X, d)$ to $(Y, e)$ is a map $f: X \rightarrow Y$ such that $d\left(x_{1}, x_{2}\right) \leq e\left(f\left(x_{1}\right), f\left(x_{2}\right)\right)$ for all $x_{1}, x_{2} \in X$. The forgetful functor $p$ : $\operatorname{Rel}_{\mathcal{V}} \rightarrow$ Set is a CLat $\wedge$-fibration, where reindexing along $f: X \rightarrow Y$ is given by $f^{*}(Y, e)=(X, e \circ f \times f)$.

For $\mathcal{V}=2=\{0,1\}$ with the usual order $0 \leq 1$, Rel $\mathcal{V}$ coincides with Rel. Another example is given by the closed interval $\mathcal{V}=[0,1]$, with the reverse order. Then, a morphism from $(X, d)$ to $(Y, e)$ is a non-expansive map $f: X \rightarrow Y$, that is, s.t. $e\left(f\left(x_{1}\right), f\left(x_{2}\right)\right) \leq d\left(x_{1}, x_{2}\right)$ (with $\leq$ the usual order, i.e., where 0 is the smallest). This instance will be denoted by $\operatorname{Rel}_{[0,1]}$.

Liftings and Coinductive Predicates. Let $p: \mathcal{E} \rightarrow \mathcal{C}$ be a fibration, and $B: \mathcal{C} \rightarrow \mathcal{C}$ a functor. A functor $\bar{B}: \mathcal{E} \rightarrow \mathcal{E}$ is called a lifting of $B$ if $p \circ \bar{B}=B \circ p$. In that case, $\bar{B}$ restricts to a functor $\bar{B}_{X}: \mathcal{E}_{X} \rightarrow \mathcal{E}_{B X}$, for any $X$ in $\mathcal{C}$.

A lifting $\bar{B}$ of $B$ gives rise to an abstract notion of coinductive predicate, as follows. For any $B$-coalgebra $(X, \gamma)$ there is the functor, i.e., monotone function defined by $\gamma^{*} \circ \bar{B}_{X}: \mathcal{E}_{X} \rightarrow$ $\mathcal{E}_{X}$. We think of post-fixed points of $\gamma^{*} \circ \bar{B}_{X}$ as invariants, generalising bisimulations. If $p$ is a CLat ${ }_{\wedge}$-fibration, then $\gamma^{*} \circ \bar{B}_{X}$ has a greatest fixed point $\nu\left(\gamma^{*} \circ \bar{B}_{X}\right)$, which is also the greatest post-fixed point. It is referred to as the coinductive predicate defined by $\bar{B}$ on $\gamma$.

Example 2.7. First, for a Set functor $B$ : Set $\rightarrow$ Set, recall the lifting $\operatorname{Rel}(B)$ of $B$ defined in the beginning of this section. We refer to $\operatorname{Rel}(B)$ as the canonical relation lifting of $B$. For a coalgebra $(X, \gamma)$, a post-fixed point of the operator $\gamma^{*} \circ \operatorname{Rel}(B)_{X}$ is a bisimulation, as explained above. The coinductive predicate $\nu\left(\gamma^{*} \circ \operatorname{Rel}(B)_{X}\right)$ defined by $\operatorname{Rel}(B)$ is bisimilarity. Another example is given by the lifting $\bar{B}$ for similarity defined in the beginning of this section, which we further study in Section 4. In that section we also define a unary predicate, divergence, making use of the predicate fibration. Coinductive predicates in the fibration $\operatorname{Re}_{[0,1]}$ can be thought of as behavioural distances, providing a quantitative analogue of bisimulations, measuring the distances between states. A simple example on deterministic automata is studied in Section 4.1.

Remark 2.8. In quantitative examples one often works in a category with more structure, e.g., by replacing $\operatorname{Rel}_{[0,1]}$ by the category of pseudo-metrics and non-expansive maps. Similarly, one can replace Rel by the category of equivalence relations. Defining liftings then requires slightly more work, and since we use fibrations to define coinductive predicates, this is not needed. Therefore, we do not use such categories in our examples.

We sometimes need the notion of fibration map: if $\bar{B}$ is a lifting of $B$, the pair $(\bar{B}, B)$ is called a fibration map if $(B f)^{*} \circ \bar{B}_{Y}=\bar{B}_{X} \circ f^{*}$ for any arrow $f: X \rightarrow Y$ in $\mathcal{C}$. If $B$ preserves weak pullbacks, then $(\operatorname{Rel}(B), B)$ is a fibration map [Jac16] in the relation fibration (Example 2.5). 
2.2. Coalgebraic Modal Logic. We recall a general duality-based approach to coalgebraic modal logic where we work in the context of a contravariant adjunction [PMW06, Kli07, JS09] in contrast to earlier work [KKP04, BK05b] that assumed a dual equivalence.

We assume the following setting, involving an adjunction $P \dashv Q$ and a natural transformation $\delta: B Q \Rightarrow Q L$ :

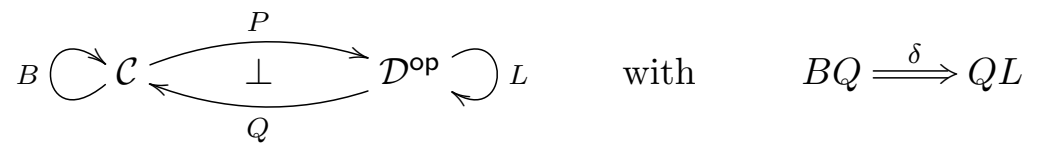

In this context, a logic for $B$-coalgebras is a pair $(L, \delta)$ as above. The functor $L: \mathcal{D} \rightarrow \mathcal{D}$ represents the syntax of the modalities. It is assumed to have an initial algebra $\alpha: L \Phi \cong$ $\Phi$, which represents the set (or other structure) of formulas of the logic. The natural transformation $\delta$ gives the one-step semantics. It can equivalently be presented in terms of its mate $\widehat{\delta}: L P \Rightarrow P B$, which is perhaps more common in the literature. However, we will formulate adequacy and expressiveness in terms of the current presentation of $\delta$.

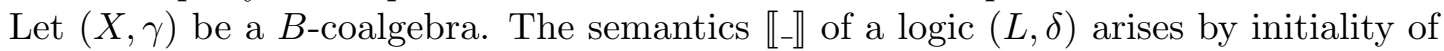
$\alpha$, making use of the mate $\widehat{\delta}$, as the unique map making the diagram on the left below commute.
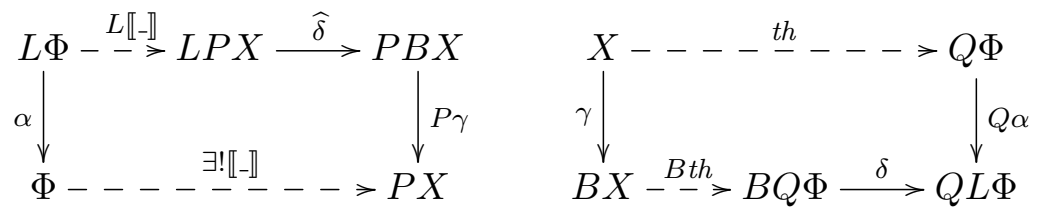

The theory map th: $X \rightarrow Q \Phi$ is defined as the transpose of $\llbracket-\rrbracket$, i.e., $t h=Q \llbracket-\rrbracket \circ \eta_{X}$ where $\eta$ : Id $\rightarrow Q P$ is the unit of the adjunction $P \dashv Q$. It is the unique map making the diagram on the right above commute.

Example 2.9. Let $\mathcal{C}=\mathcal{D}=$ Set, $P=Q=2^{-}$the contravariant powerset functor, and $B X=2 \times X^{A}$. We define a simple logic for $B$-coalgebras, where formulas are just words over $A$. To this end, let $L X=A \times X+1$. The initial algebra of $L$ is the set $A^{*}$ of words. Define $\delta: B Q \Rightarrow Q L$ on a component $X$ as follows:

$$
\delta_{X}: 2 \times\left(2^{X}\right)^{A} \rightarrow 2^{A \times X+1} \quad \delta_{X}(o, t)(u)= \begin{cases}o & \text { if } u=* \in 1 \\ t(a)(x) & \text { if } u=(a, x) \in A \times X\end{cases}
$$

For a coalgebra $\langle o, t\rangle: X \rightarrow 2 \times X^{A}$, the associated theory map th: $X \rightarrow 2^{A^{*}}$ is given by $\operatorname{th}(x)(\varepsilon)=o(x)$ and $\operatorname{th}(x)(a w)=\operatorname{th}(t(x)(a))(w)$ for all $x \in X, a \in A, w \in A^{*}$. This is, of course, the usual semantics of deterministic automata.

In the above example, the logic does not contain propositional connectives; this is reflected by the choice $\mathcal{D}=$ Set. Although it is possible to include propositional connectives into the functor $L$ (cf. e.g. [Kli07]), one usually adds those connectives by choosing $\mathcal{D}$ to be a category of algebras. For instance, Boolean algebras are a standard choice for propositional logic, and in Section 4 we use the category of semilattices to represent conjunction. In fact, if one is only interested in defining the semantics of the logic, one can simply work with algebras for a signature; this is supported by the adjunctions presented in the next subsection. We outline in the next subsection how this can be used to represent the propositional part of a real-valued modal logic. 
2.3. Contravariant Adjunctions. In this subsection we discuss several adjunctions that we use for presenting coalgebraic logic as above, and will allow us in Section 4 to demonstrate that a large variety of concrete examples is covered by our framework. In all cases, the adjunctions that we use for the logic are generated by an object $\Omega$ of 'truth values'. In fact, we believe all of the dual adjunctions listed in this section are instances of the so-called concrete dualities from [PT91] where $\Omega$ is the dualising object inducing the adjunction.

For a simple but useful class of such adjunctions, let $\mathcal{D}$ be a category with products, and $\Omega$ an object in $\mathcal{D}$. Then there is an adjunction

$$
P \dashv Q \text { : Set } \leftrightarrows \mathcal{D}^{\text {op }} \quad \text { where } P X=\Omega^{X} \text { and } Q X=\operatorname{Hom}(X, \Omega),
$$

where $\Omega^{X}$ is the $X$-fold product of $\Omega$.

This adjunction is instrumental for representing the semantics of a coalgebraic modal logic for $B$-coalgebras based on predicate liftings (cf. e.g. [KP11]) within the dual adjunction framework by defining a suitable category of $L$-algebras. In general, describing the category of $L$-algebras that precisely represents a given logic (i.e., where the initial algebra corresponds to the set of formulas modulo equivalence) is nontrivial. For studying expressiveness, however, it is sufficient to consider formulas and their semantics. This can be done as follows: We start by considering a set $\mathcal{O}$ of propositional operators, each $o \in \mathcal{O}$ associated with a certain finite arity $\operatorname{ar}(o) \in \mathbb{N}$ and define the (propositional) signature functor

$$
\Sigma_{\mathcal{O}}: \text { Set } \rightarrow \text { Set } \quad \text { by putting } \quad \Sigma_{\mathcal{O}} X:=\coprod_{o \in \mathcal{O}} X^{\operatorname{ar}(o)} .
$$

The category $\operatorname{Alg}\left(\Sigma_{\mathcal{O}}\right)$ of algebras for the functor $\Sigma_{\mathcal{O}}$ will play the role of the category $\mathcal{D}$ in (2.4). We assume that we are given a set of truth values $\Omega$ together with a $\Sigma_{\mathcal{O}}$-algebra structure $a_{\Omega}: \Sigma_{\mathcal{O}} \Omega \rightarrow \Omega$, which gives an interpretation of the propositional operators. As $\Omega$ is a $\Sigma_{\mathcal{O}}$-algebra we obtain functors $P$ : Set $\rightarrow \operatorname{Alg}\left(\Sigma_{\mathcal{O}}\right)^{\text {op }}$ and $Q: \operatorname{Alg}\left(\Sigma_{\mathcal{O}}\right)^{\text {op }} \rightarrow$ Set as described in (2.4). An $\Omega$-valued coalgebraic modal logic $\mathcal{L}(\Lambda)$ for a functor $B$ : Set $\rightarrow$ Set is now given as a set $\Lambda$ of modal operators where each $\lambda \in \Lambda$ is an $\Omega$-valued predicate lifting $\lambda: P^{n} \Rightarrow P B$ with $\operatorname{ar}(\lambda)=n$ the arity of $\lambda$. Given $\mathcal{L}(\Lambda)$ we define $L_{\Lambda}: \operatorname{Alg}\left(\Sigma_{\mathcal{O}}\right) \rightarrow \operatorname{Alg}\left(\Sigma_{\mathcal{O}}\right)$ by putting

$$
L_{\Lambda} A:=T_{\Sigma_{\mathcal{O}}}\left(\left\{[\lambda]\left(a_{1}, \ldots, a_{n}\right) \mid \lambda \in \Lambda, n=\operatorname{ar}(\lambda), a_{j} \in A \text { for } 1 \leq j \leq n\right\}\right)
$$

where $[\lambda]\left(a_{1}, \ldots, a_{n}\right)$ should be understood as name of a generator and where $T_{\Sigma_{\mathcal{O}}}$ denotes the free (term) monad over $\Sigma_{\mathcal{O}}$. The action of $L_{\Lambda}$ on a given morphism $f: A \rightarrow B$ is defined to be the unique $\operatorname{Alg}\left(\Sigma_{\mathcal{O}}\right)$-morphism extending the map $\left[\lambda_{i}\right]\left(a_{1}, \ldots, a_{n}\right) \mapsto\left[\lambda_{i}\right]\left(f\left(a_{1}\right), \ldots, f\left(a_{n}\right)\right)$. It is now easy to see that the predicate liftings in $\Lambda$ give rise to a natural transformation $\widehat{\delta}: L_{\Lambda} P \Rightarrow P B$ where, for an arbitrary set $X$, the $X$ component $\widehat{\delta}_{X}: L_{\Lambda} P X \rightarrow P B X$ is the unique extension of the map

$$
[\lambda]\left(u_{1}, \ldots, u_{n}\right) \mapsto \lambda\left(u_{1}, \ldots, u_{n}\right)
$$

for $\lambda \in \Lambda, n=\operatorname{ar}(\lambda)$ and $u_{j} \in \Omega^{X}$ for $1 \leq j \leq n$. In other words, $\left(L_{\Lambda}, \delta\right)$ with $\delta$ being the mate of $\widehat{\delta}$ is a logic for $B$-coalgebras in the sense of (2.3). We arrive at the following picture:

$$
B C \text { Set } \underset{Q=\operatorname{Hom}(-, \Omega)}{\frac{P=\Omega^{-}}{\perp}} \operatorname{Llg}\left(\Sigma_{\mathcal{O}}\right)^{\mathrm{op}} \bigcirc L_{\Lambda} \quad \delta: B Q \Rightarrow Q L_{\Lambda}
$$


Example 2.10. To illustrate the outlined approach, consider the real-valued coalgebraic modal logics from [KM18]. The set $\Phi$ of formulas of these logics is given by the following definition that is indexed by a set $\Lambda$ of unary modal operators:

$$
\Phi::=\top|[\lambda] \varphi, \lambda \in \Lambda| \min \left(\varphi_{1}, \varphi_{2}\right)|\neg \varphi| \varphi \ominus q, q \in \mathbb{Q} \cap[0, \top]
$$

where $[0, \top]$ is a closed interval of real numbers with $\top$ denoting an arbitrary positive real number, $\ominus$ is interpreted as truncated subtraction on $[0, \top]$ given by $p \ominus q:=\max (p-q, 0)$, min is interpreted as minimum and negation on $[0, \top]$ is defined as $\neg q:=\top-q$. Following the construction of $L_{\Lambda}$ as described above, we obtain the following dual adjunction:

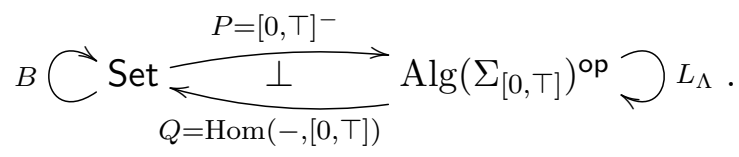

Here the operations on $[0, \top]$ are $\top$, min, $\neg$ and $-\ominus q$ for $q \in \mathbb{Q} \cap[0, \top]$, thus

$$
\Sigma_{[0, \top]} X=1+X^{2}+X+X \times(\mathbb{Q} \cap[0, \top]) \quad \text { and } \quad L_{\Lambda}(A)=T_{\Sigma_{[0, \top]}}(\{[\lambda] a \mid a \in A, \lambda \in \Lambda\}) .
$$

To study expressiveness relative to a coinductive predicate in a fibration $p: \mathcal{E} \rightarrow \mathcal{C}$ we rely on a given dual adjunction $P \dashv Q$ between $\mathcal{C}$ and $\mathcal{D}$ together with its lifted version $\bar{P} \dashv \bar{Q}$ between $\mathcal{E}$ and $\mathcal{D}$. In a large class of examples the fibration under consideration will be of type $p: \operatorname{Rel} \mathcal{V} \rightarrow$ Set with $P \dashv Q$ being the dual adjunction between Set and $\operatorname{Alg}(\Sigma)$ described above. We will now provide a proposition that yields the required dual adjunction $\bar{P} \dashv \bar{Q}$ between $\operatorname{Rel}_{\mathcal{V}}$ and $\operatorname{Alg}(\Sigma)$. To obtain this dual adjunction we need a number of assumptions. First we make some assumptions on the truth and distance values $\Omega$ and $\mathcal{V}$ :

- $\mathcal{V}$ is a complete lattice of distance values,

- $\Omega$ is a bounded poset of truth values,

- $\left(\Omega, R_{\Omega}: \Omega \times \Omega \rightarrow \mathcal{V}\right) \in \operatorname{Rel}_{\mathcal{V}}$.

Furthermore we let $\Delta:$ Set $\rightarrow \operatorname{Rel}_{\mathcal{V}}$ be the diagonal functor given by $\Delta X=\Delta_{X}$ where

$$
\Delta_{X}\left(x_{1}, x_{2}\right):= \begin{cases}\top & \text { if } x_{1}=x_{2} \\ \perp & \text { otherwise. }\end{cases}
$$

Proposition 2.11. Let $\Omega$ and $\mathcal{V}$ be sets of truth and distance values that satisfy the above assumptions and let $\Sigma$ : Set $\rightarrow$ Set be a functor. Suppose furthermore that $\Sigma$ has a lifting $\bar{\Sigma}:$ Rel $\mathcal{V} \rightarrow \operatorname{Rel} \mathcal{V}$ such that (i) $\Delta \circ \Sigma \leq \bar{\Sigma} \circ \Delta$ and (ii) for any $(X, R),(Y, S) \in \operatorname{Rel}_{\mathcal{V}}$ there is a morphism $\overline{\mathrm{st}}_{R, S}: R \times \bar{\Sigma} S \rightarrow \bar{\Sigma}(R \times S)$ above the strength map st $_{X, Y}: X \times \Sigma Y \rightarrow \Sigma(X \times Y)$ (the latter exists for any set functor $\Sigma$ ). If $\bar{\Sigma} R_{\Omega} \leq a_{\Omega}^{*}\left(R_{\Omega}\right)$, then there is a dual adjunction

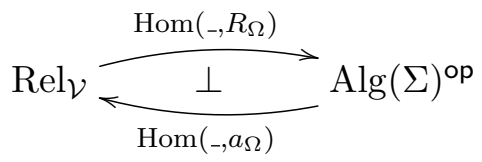

Proof. We first have to show that the functors that form the adjunction are well-defined. In the following we write $\alpha$ as abbreviation for an algebra $(A, \alpha)$. Throughout this proof we denote the least and the largest element of $\mathcal{V}$ by $\perp$ and $T$, respectively. Recall that the

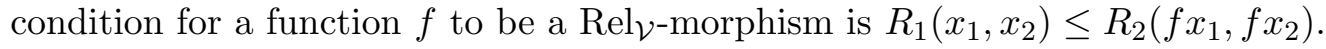


To see that the functors are well-defined on objects, first note that for each $\Sigma$-algebra $(A, \alpha)$, the set $\operatorname{Hom}\left(\alpha, a_{\Omega}\right)$ can be turned into a Rel $\mathcal{V}$-object by defining

$$
\begin{aligned}
R_{\operatorname{Hom}\left(\alpha, a_{\Omega}\right)}: \operatorname{Hom}\left(\alpha, a_{\Omega}\right) \times \operatorname{Hom}\left(\alpha, a_{\Omega}\right) & \rightarrow \mathcal{V} \\
\left(h_{1}, h_{2}\right) & \mapsto \bigwedge_{a \in A} R_{\Omega}\left(h_{1}(a), h_{2}(a)\right) .
\end{aligned}
$$

Likewise, for each $\operatorname{Rel}_{\mathcal{V}}$-object $(X, R)$, the set $\operatorname{Hom}\left(R, R_{\Omega}\right)$ carries a $\Sigma$-algebra structure $a_{R}: \Sigma \operatorname{Hom}\left(R, R_{\Omega}\right) \rightarrow \operatorname{Hom}\left(R, R_{\Omega}\right)$ given by the function that maps $t: 1 \rightarrow \Sigma \operatorname{Hom}\left(R, R_{\Omega}\right)$ to the following composition of arrows:

$$
R \cong R \times 1 \stackrel{\mathrm{id} \times t}{\longrightarrow} R \times \Delta \Sigma D \stackrel{\leq}{\longrightarrow} R \times \bar{\Sigma} \Delta D \stackrel{\overline{\mathrm{st}}_{R, \Delta D}}{\longrightarrow} \bar{\Sigma}(R \times \Delta D) \stackrel{\bar{\Sigma} \mathrm{ev}}{\longrightarrow} \bar{\Sigma} R_{\Omega} \stackrel{\overline{a_{\Omega}}}{\longrightarrow} R_{\Omega}
$$

where $D=\operatorname{Hom}\left(R, R_{\Omega}\right)$ and where $\operatorname{ev}(x, f)=f(x)$ is the evaluation function. To see that the above is a well-defined $\operatorname{Rel}_{V}$-morphism we only have to check that ev $\in$ Rel $_{\mathcal{V}}$ as the other arrows are morphisms by our assumption that $\Sigma$ has a lifting $\bar{\Sigma}: \operatorname{Rel}_{\mathcal{V}} \rightarrow \operatorname{Rel}_{\mathcal{V}}$ such that st and $a_{\Omega}$ become morphisms in Relv. We now show that ev satisfies the Rel $\mathcal{V}$ morphism condition. Consider two pairs $\left(x_{1}, f_{1}\right),\left(x_{2}, f_{2}\right) \in X \times \operatorname{Hom}\left(R, R_{\Omega}\right)$. We distinguish cases:

Case: $f_{1}=f_{2}=f$ In this case we have

$$
\begin{aligned}
R \times \Delta D\left(\left(x_{1}, f\right),\left(x_{2}, f\right)\right) & =R\left(x_{1}, x_{2}\right) \wedge \top=R\left(x_{1}, x_{2}\right) \\
& \leq R_{\Omega}\left(f\left(x_{1}\right), f\left(x_{2}\right)\right)=R_{\Omega}\left(\operatorname{ev}\left(x_{1}, f\right), \operatorname{ev}\left(x_{2}, f\right)\right) .
\end{aligned}
$$

Case: $f_{1} \neq f_{2}$. Then

$$
R \times \Delta D\left(\left(x_{1}, f_{1}\right),\left(x_{2}, f_{2}\right)\right)=R\left(x_{1}, x_{2}\right) \wedge \perp=\perp \leq R_{\Omega}\left(\operatorname{ev}\left(x_{1}, f_{1}\right), \operatorname{ev}\left(x_{2}, f_{2}\right)\right) .
$$

To see that the Hom-functors are well-defined on morphisms we first check that $\operatorname{Hom}\left(-, a_{\Omega}\right)$ maps algebra morphisms to morphisms in Rel $\mathcal{V}$. To this aim consider an algebra morphism $h:\left(A_{1}, \alpha_{1}\right) \rightarrow\left(A_{2}, \alpha_{2}\right)$ and $g_{1}, g_{2} \in \operatorname{Hom}\left(\alpha_{2}, a_{\Omega}\right)$. We calculate:

$$
\begin{aligned}
R_{\mathrm{Hom}\left(\alpha_{2}, a_{\Omega}\right)}\left(g_{1}, g_{2}\right) & =\bigwedge_{a \in A_{2}} R_{\Omega}\left(g_{1}(a), g_{2}(a)\right) \leq \bigwedge_{a^{\prime} \in A_{1}} R_{\Omega}\left(g_{1}\left(h\left(a^{\prime}\right)\right), g_{2}\left(h\left(a^{\prime}\right)\right)\right) \\
& =R_{\operatorname{Hom}\left(\alpha_{1}, a_{\Omega}\right)}\left(\operatorname{Hom}\left(h, a_{\Omega}\right)\left(g_{1}\right), \operatorname{Hom}\left(h, a_{\Omega}\right)\left(g_{2}\right)\right)
\end{aligned}
$$

We now check that the functor $\operatorname{Hom}\left(, R_{\Omega}\right)$ is well-defined on morphisms as well. Let $h:\left(X_{1}, R_{1}\right) \rightarrow\left(X_{2}, R_{2}\right) \in \operatorname{Rel}_{\mathcal{V}}$. We have to show that $\operatorname{Hom}\left(h, R_{\Omega}\right): \operatorname{Hom}\left(R_{2}, R_{\Omega}\right) \rightarrow$ $\operatorname{Hom}\left(R_{1}, R_{\Omega}\right)$ is a $\Sigma$-algebra morphism.

We calculate:

$$
\begin{array}{rll}
\operatorname{Hom}\left(h, R_{\Omega}\right)\left(a_{\operatorname{Hom}\left(R_{2}, R_{\Omega}\right)}(t)\right) & = & \operatorname{Hom}\left(h, R_{\Omega}\right)\left(\lambda x \cdot a_{\Omega} \circ \Sigma \mathrm{ev} \circ \operatorname{st}(x, t)\right) \\
= & \lambda y \cdot a_{\Omega} \circ \Sigma \mathrm{ev} \circ \operatorname{st}(h(y), t) \\
\stackrel{\text { nat. of st }}{=} & \lambda y \cdot a_{\Omega} \circ \Sigma \mathrm{ev} \circ \Sigma(h \times \mathrm{id}) \circ \operatorname{st}(y, t) \\
\stackrel{(*)}{=} & \lambda y \cdot a_{\Omega} \circ \Sigma \mathrm{ev} \circ \Sigma\left(\mathrm{id} \times \operatorname{Hom}\left(h, R_{\Omega}\right)\right) \circ \operatorname{st}(y, t) \\
\stackrel{\text { nat. of st }}{=} & \lambda y \cdot a_{\Omega} \circ \Sigma \mathrm{ev} \circ \operatorname{st}\left(y, \Sigma \operatorname{Hom}\left(h, R_{\Omega}\right)(t)\right) \\
\stackrel{\text { Def. of } a_{\operatorname{Hom}(\ldots)}^{=}}{ } & a_{\operatorname{Hom}\left(R_{1}, R_{\Omega}\right)}\left(\Sigma \operatorname{Hom}\left(h, R_{\Omega}\right)(t)\right)
\end{array}
$$


where $\left(^{*}\right)$ holds as the following diagram can be easily seen to commute in Set:

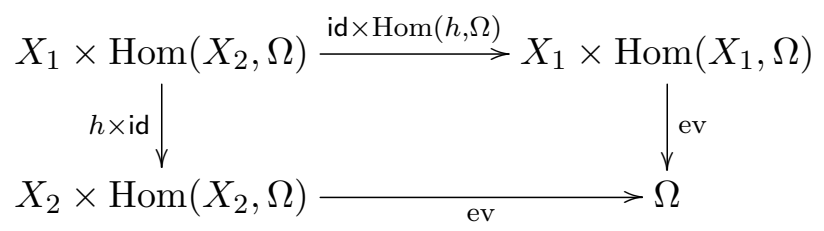

This finishes the argument that the functors are well-defined. We will now argue that they form an adjunction. We prove this by defining the unit and counit of the adjunction satisfying the triangle identities.

For $(X, R) \in \operatorname{Rel}_{\mathcal{V}}$ we define the unit map $\eta_{R}: R \rightarrow \operatorname{Hom}\left(\operatorname{Hom}\left(R, R_{\Omega}\right), a_{\Omega}\right)$ by putting $\eta_{R}(x):=\lambda f . f(x)$. Naturality of $\eta$ can be easily checked (left to the reader), but welldefinedness is not obvious. For the latter we have to show that $\eta_{R}$ is a Rel $\mathcal{V}$-morphism and that $\eta_{R}(x)$ is a $\operatorname{Alg}(\Sigma)$-morphism for all $(X, R) \in \operatorname{Rel}_{\mathcal{V}}$ and all $x \in X$.

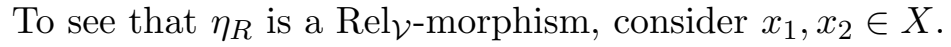

$$
\begin{aligned}
R\left(x_{1}, x_{2}\right) & \leq \bigwedge_{f \in \operatorname{Hom}\left(R, R_{\Omega}\right)} R_{\Omega}\left(f\left(x_{1}\right), f\left(x_{2}\right)\right)=\bigwedge_{f \in \operatorname{Hom}\left(R, R_{\Omega}\right)} R_{\Omega}\left(\eta_{R}\left(x_{1}\right)(f), \eta_{R}\left(x_{2}\right)(f)\right) \\
& =R_{\operatorname{Hom}\left(\operatorname{Hom}\left(R, R_{\Omega}\right), a_{\Omega}\right)}\left(\eta_{R}\left(x_{1}\right), \eta_{R}\left(x_{2}\right)\right)
\end{aligned}
$$

To check that $\eta_{R}(x)$ is a $\Sigma$-algebra morphism, we calculate

$$
\begin{aligned}
\left(a_{\Omega} \circ \Sigma \eta_{R}(x)\right)(t) & =\left(a_{\Omega} \circ \Sigma(\lambda f \cdot f(x))(t)=\left(a_{\Omega} \circ \Sigma\left(\mathrm{ev}\left(x,,_{-}\right)\right)\right)(t)\right. \\
& =\left(a_{\Omega} \circ \Sigma(\mathrm{ev} \circ \mathrm{st})\right)(x, t) \\
& =\eta_{R}(x)\left(\lambda x .\left(a_{\Omega} \circ \Sigma(\mathrm{ev} \circ \mathrm{st})\right)(x, t)\right)=\eta_{R}(x)\left(a_{\mathrm{Hom}\left(R, R_{\Omega}\right)}(t)\right)
\end{aligned}
$$

For the counit of the (dual) adjunction we define $\epsilon_{\alpha}:(A, \alpha) \rightarrow \operatorname{Hom}\left(\operatorname{Hom}\left(\alpha, a_{\Omega}\right), R_{\Omega}\right)$ by putting $\epsilon_{\alpha}(a):=\lambda g . g(a)$ for all $(A, \alpha) \in \operatorname{Alg}(\Sigma)$ and all $a \in A$. Again we leave it to the reader to convince themselves that $\epsilon$ is natural. We have to check well-definedness, i.e, we need to check that $\epsilon_{\alpha}(a)$ is a $\operatorname{Rel} \mathcal{V}$-morphism and that $\epsilon_{\alpha}$ is an $\operatorname{Alg}(\Sigma)$-morphism.

To see that $\epsilon_{\alpha}(a)$ is a $\operatorname{Rel}_{\mathcal{V}}$-morphism we consider $g_{1}, g_{2} \in \operatorname{Hom}\left(\alpha, a_{\Omega}\right)$ :

$$
\begin{aligned}
R_{\mathrm{Hom}\left(\alpha, a_{\Omega}\right)}\left(g_{1}, g_{2}\right) & =\bigwedge_{a^{\prime} \in A} R_{\Omega}\left(g_{1}\left(a^{\prime}\right), g_{2}\left(a^{\prime}\right)\right) \leq R_{\Omega}\left(g_{1}(a), g_{2}(a)\right) \\
& =R_{\Omega}\left(\epsilon_{\alpha}(a)\left(g_{1}\right), \epsilon_{\alpha}(a)\left(g_{2}\right)\right)
\end{aligned}
$$

To check that $\epsilon_{\alpha}$ is an $\operatorname{Alg}(\Sigma)$-morphism we calculate:

$$
\begin{array}{cll}
a_{\mathrm{Hom}\left(\operatorname{Hom}\left(\alpha, a_{\Omega}\right), R_{\Omega}\right)} \circ \Sigma \epsilon_{\alpha}(t) & = & \left(\lambda g .\left(a_{\Omega} \circ \Sigma \mathrm{ev} \circ \mathrm{st}\right)\left(g, \Sigma \epsilon_{\alpha}(t)\right)\right. \\
\stackrel{\text { Def. of st }^{=}}{=} & \left(\lambda g \cdot\left(a_{\Omega} \circ \Sigma \mathrm{ev}\left(g,{ }_{-}\right)\right)\right)\left(\Sigma \epsilon_{\alpha}(t)\right) \\
\stackrel{(+)}{=} & \left(\lambda g \cdot\left(a_{\Omega} \circ \Sigma g\right)\right)(t) \\
& \stackrel{g \text { alg. mor. }}{=} & \lambda g \cdot g(\alpha(t))=\epsilon_{\alpha}(\alpha(t))
\end{array}
$$

where $(+)$ is an easy consequence of $\left(\operatorname{ev}\left(g,{ }_{-}\right) \circ \epsilon_{\alpha}\right)(a)=g(a)$ for all $a \in A$.

This finishes the definition of unit and counit of the adjunction - checking the triangle equalities is a straightforward exercise.

The following remark is obvious, but at the same time useful for concrete examples. 
Remark 2.12. Let $\mathcal{C}$ be a full subcategory of $\operatorname{Rel} \mathcal{V}$ and $\mathcal{D}$ a full subcategory of $\operatorname{Alg}(\Sigma)$ such that $\operatorname{Hom}\left(-, a_{\Omega}\right)$ and $\operatorname{Hom}\left(-, R_{\Omega}\right)$ restrict to functors of type $\mathcal{D} \rightarrow \mathcal{C}$ and of type $\mathcal{C} \rightarrow \mathcal{D}$, respectively. Then the dual adjunction from Prop. 2.11 restricts to a dual adjunction between $\mathcal{C}$ and $\mathcal{D}$.

The assumptions in Proposition 2.11 concerning existence of a suitable lifting of $\Sigma$ are in particular met when $\Sigma$ is a polynomial functor.

Corollary 2.13. Let $\Omega$ and $\mathcal{V}$ be sets of truth and distance values that satisfy the assumptions from Prop. 2.11, let $\Sigma$ be a signature functor. Then $\Sigma$ lifts to Rel $\mathcal{V}$ such that (i) $\Delta \circ \Sigma \leq \bar{\Sigma} \circ \Delta$ and (ii) there is a strength map $\overline{\mathrm{st}}_{R, S}$ above $\mathrm{st}_{X, Y}$. Consequently, the dual adjunction $\operatorname{Hom}\left({ }_{-}, R_{\Omega}\right) \dashv \operatorname{Hom}\left({ }_{-}, a_{\Omega}\right)$ from (2.7) $\operatorname{Hom}\left({ }_{-}, R_{\Omega}\right) \dashv \operatorname{Hom}\left(a_{\Omega},{ }_{-}\right)$exists if $\bar{\Sigma} R_{\Omega} \leq a_{\Omega}^{*}\left(R_{\Omega}\right)$.

Proof. It is clear that the existence of the dual adjunction follows from Prop 2.11 once we establish that any polynomial functor $\Sigma$ has a lifting to $\operatorname{Rel}_{\mathcal{V}}$ such that (i) $\Delta \circ \Sigma \leq \bar{\Sigma} \circ \Delta$ and (ii) there is a strength map $\overline{\operatorname{st}}_{R, S}: R \times \bar{\Sigma} S \rightarrow \bar{\Sigma}(R \times S)$ above st ${ }_{X, Y}$ for all $R \in\left(\operatorname{Rel}_{\mathcal{V}}\right)_{X}, S \in$ $(\operatorname{Rel} \mathcal{V})_{Y}$. In the following we prove this claim not only for signature functors but for the collection of functors $\mathcal{F}$ generated by the following grammar:

$$
\mathcal{F}::=A \in \text { Set }|\mathrm{Id}| \prod_{j \in J} \Sigma_{j} \mid \coprod_{j \in J} \Sigma_{j}
$$

where $J$ are arbitrary sets of indexes, $A$ denotes the constant functor and Id: Set $\rightarrow$ Set denotes the identity functor. For a functor $\Sigma \in \mathcal{F}$ we now inductively define the action of its lifting $\bar{\Sigma}: \operatorname{Rel}_{\mathcal{V}} \rightarrow \operatorname{Rel}_{\mathcal{V}}$ on objects while at the same time proving conditions (i) and (ii).

Case: $\Sigma=A$ (constant functor). Then we put

$$
(\bar{A} R)\left(a_{1}, a_{2}\right):=\left\{\begin{array}{cc}
\top & \text { if } a_{1}=a_{2} \\
\perp & \text { otherwise. }
\end{array}\right.
$$

The conditions on $\Delta$ and st are easy to check as in this case $\Delta A X=\Delta_{A}=\bar{A} \Delta X$ and as $\operatorname{st}_{X, Y}(x, a)=a$ which clearly lifts to a suitable $\overline{\mathrm{st}}_{R, S}$.

Case: $\Sigma=$ Id. Then $\bar{\Sigma}(R)=R, \Delta \circ \Sigma=\Delta=\bar{\Sigma} \circ \Delta$ and the strength map is simply the identity.

Case: $\Sigma=\prod_{j \in J} \Sigma_{j}$. Then

$$
\bar{\Sigma}(R)\left(x_{1}, x_{2}\right)=\bigwedge_{j \in J} \bar{\Sigma}_{j}(R)\left(\pi_{j}\left(x_{1}\right), \pi_{j}\left(x_{2}\right)\right)
$$

where $\pi_{j}$ is the projection onto the $j$-th component of the product. For proving property (i) we consider an arbitrary set $X, x_{1}, x_{2} \in \Sigma X$ and we calculate:

$$
\begin{aligned}
\Delta \Sigma X\left(x_{1}, x_{2}\right) & =\Delta \prod_{j \in J} \Sigma_{j} X\left(x_{1}, x_{2}\right)=\bigwedge_{j \in J} \Delta\left(\Sigma_{j} X\right)\left(\pi_{j}\left(x_{1}\right), \pi_{j}\left(x_{2}\right)\right) \\
& \stackrel{\text { I.H. }}{\leq} \bigwedge_{j \in J} \bar{\Sigma}_{j}(\Delta X)\left(\pi_{j}\left(x_{1}\right), \pi_{j}\left(x_{2}\right)\right)=\bar{\Sigma}(\Delta X)\left(x_{1}, x_{2}\right)
\end{aligned}
$$


To check that st: $X \times \Sigma Y \rightarrow \Sigma(X \times Y)$ satisfies condition (ii) let st s $_{j}: X \times \Sigma_{j} Y \rightarrow$ $\Sigma_{j}(X \times Y)$ and consider two arbitrary pairs $\left(x_{1}, z_{1}\right),\left(x_{2}, z_{2}\right) \in X \times \Sigma Y$. We calculate:

$$
\begin{aligned}
(R \times \bar{\Sigma} S)\left(\left(x_{1}, z_{1}\right),\left(x_{2}, z_{2}\right)\right) & =R\left(x_{1}, x_{2}\right) \wedge \bar{\Sigma} S\left(z_{1}, z_{2}\right) \\
& =\bigwedge\left\{R\left(x_{1}, x_{2}\right)\right\} \cup\left\{\bar{\Sigma}_{j} S\left(\pi_{j}\left(z_{1}\right), \pi_{j}\left(z_{2}\right)\right) \mid j \in J\right\} \\
& =\bigwedge_{j \in J}\left\{\left(R \times \bar{\Sigma}_{j} S\right)\left(\left(x_{1}, \pi_{j}\left(z_{1}\right)\right),\left(x_{2}, \pi_{j}\left(z_{2}\right)\right)\right)\right\} \\
& \stackrel{\text { I.H. }}{\leq} \bigwedge_{j \in J}\left\{\bar{\Sigma}_{j}(R \times S)\left(\operatorname{st}_{j}\left(x_{1}, \pi_{j}\left(z_{1}\right)\right), \operatorname{st}_{j}\left(x_{2}, \pi_{j}\left(z_{2}\right)\right)\right)\right\} \\
& \stackrel{(+)}{=} \bigwedge_{j \in J}\left\{\bar{\Sigma}_{j}(R \times S)\left(\pi_{j}\left(\operatorname{st}\left(x_{1}, z_{1}\right)\right), \pi_{j}\left(\operatorname{st}\left(x_{2}, z_{2}\right)\right)\right)\right\} \\
& \stackrel{\text { Def. }}{=} \bar{\Sigma}(R \times S)\left(\operatorname{st}\left(x_{1}, z_{1}\right), \operatorname{st}\left(x_{2}, z_{2}\right)\right)
\end{aligned}
$$

where for $(+)$ we used that $\pi_{j} \circ$ st $=\mathrm{st}_{j} \circ\left(\mathrm{id} \times \pi_{j}\right)$ as can be easily checked.

Case: $\Sigma=\bigsqcup_{j \in J} \Sigma_{j}$. Then

$$
\bar{\Sigma}(R)\left(\kappa_{m}\left(x_{1}\right), \kappa_{n}\left(x_{2}\right)\right)=\left\{\begin{array}{l}
\bar{\Sigma}_{n}(R)\left(x_{1}, x_{2}\right) \text { if } n=m \\
\perp \text { otherwise. }
\end{array}\right.
$$

where the $\kappa_{n}$ denotes the $n$-th inclusion into the coproduct. As in the previous case we first verify (i): let $X$ be a set and consider $x_{1}, x_{2} \in \Sigma X$. W.l.o.g. we assume there are $j \in J$ and $x_{1}^{\prime}, x_{2}^{\prime} \in \Sigma_{j} X$ with $x_{i}=\kappa_{j}\left(x_{i}^{\prime}\right)$ for $i=1,2$ - otherwise property (i) is trivially satisfied. Spelling out the definitions we get

$$
\Delta(\Sigma X)\left(x_{1}, x_{2}\right)=\Delta\left(\Sigma_{j} X\right)\left(x_{1}^{\prime}, x_{2}^{\prime}\right) \stackrel{\text { I.H. }}{\leq} \bar{\Sigma}_{j}(\Delta X)\left(x_{1}^{\prime}, x_{2}^{\prime}\right)=\bar{\Sigma}(\Delta X)\left(x_{1}, x_{2}\right)
$$

Let st ${ }_{j}: X \times \Sigma_{j} Y \rightarrow \Sigma_{j}(X \times Y)$ be the strength maps of the components of $\Sigma$. Consider pairs $\left(x_{1}, \kappa_{j}\left(y_{1}\right)\right),\left(x_{2}, \kappa_{j}\left(y_{2}\right)\right) \in X \times \Sigma Y$ where we assumed that the $y_{i}$ 's are from the same $j$-th component of $\Sigma Y$ - otherwise the strength condition is trivially true. We calculate:

$$
\begin{aligned}
(R \times \bar{\Sigma} S)\left(\left(x_{1}, \kappa_{j}\left(y_{1}\right)\right),\left(x_{2}, \kappa_{j}\left(y_{2}\right)\right)\right) & \stackrel{\text { Def of }}{=} & \left(R \times \bar{\Sigma}_{j} S\right)\left(\left(x_{1}, y_{1}\right),\left(x_{2}, y_{2}\right)\right) \\
& \stackrel{\text { I.H. }}{\leq} & \bar{\Sigma}_{j}\left(\operatorname{st}_{j}\left(x_{1}, y_{1}\right), \mathrm{st}_{j}\left(x_{2}, y_{2}\right)\right) \\
& = & \bar{\Sigma}(R \times S)\left(\kappa_{j}\left(\operatorname{st}_{j}\left(x_{1}, y_{1}\right)\right), \kappa_{j}\left(\operatorname{st}_{j}\left(x_{2}, y_{2}\right)\right)\right) \\
& = & \bar{\Sigma}(R \times S)\left(\operatorname{st}\left(x_{1}, \kappa_{j}\left(y_{1}\right)\right), \operatorname{st}\left(x_{2}, \kappa_{j}\left(y_{2}\right)\right)\right)
\end{aligned}
$$

where the last equality follows from the easily verifiable fact that $\kappa_{j} \circ \mathrm{st}_{j}=$ sto $\left(\mathrm{id} \times \kappa_{j}\right)$. This finishes the definition of $\bar{\Sigma}$ on objects. Our argument also shows that for polynomial functors $\Sigma$, the map st lifts to Rel $\mathcal{V}$ as required. Finally, we extend $\bar{\Sigma}$ to a functor $\operatorname{Rel}_{\mathcal{V}} \rightarrow$ Rel $\mathcal{V}$ by putting $\bar{\Sigma} f:=\Sigma f$ for all morphisms $f: R \rightarrow S \in \operatorname{Rel}_{\mathcal{V}}$. In order to see that $\bar{\Sigma}$ is well defined on morphisms one has to prove that $\Sigma f$ is a $\operatorname{Rel}_{\mathcal{V}}$-morphism from $\bar{\Sigma}(R)$ to $\bar{\Sigma}(S)$ whenever $f: R \rightarrow S$ is a $\operatorname{Rel}_{\mathcal{V}}$-morphism. This can be easily shown by induction on the structure of $\Sigma$. Functoriality of $\bar{\Sigma}$ is an immediate consequence of functoriality of $\Sigma$.

Let $\mathcal{L}(\Lambda)$ be a coalgebraic modal logic for some functor $B$ and its representation via a dual adjunction from Set to $\operatorname{Alg}\left(\Sigma_{\mathcal{O}}\right)$ for some polynomial functor $\Sigma_{\mathcal{O}}$ together with a functor $L_{\Lambda}: \operatorname{Alg}\left(\Sigma_{\mathcal{O}}\right) \rightarrow \operatorname{Alg}\left(\Sigma_{\mathcal{O}}\right)$ as in (2.6). Furthermore let $a_{\Omega}: \Sigma_{\mathcal{O}} \Omega \rightarrow \Omega$ be the algebra 
structure induced by the propositional operators $\mathcal{O}$ of $\mathcal{L}(\Lambda)$ such that for all operations $o \in O$ and all $v_{1}, \ldots, v_{n}, v_{1}^{\prime}, \ldots, v_{n}^{\prime} \in \Omega$ we have

$$
\bigwedge_{1 \leq i \leq n} R_{\Omega}\left(v_{i}, v_{i}^{\prime}\right) \leq R_{\Omega}\left(o\left(v_{1}, \ldots, v_{n}\right), o\left(v_{1}^{\prime}, \ldots, v_{n}^{\prime}\right)\right)
$$

Then the results of this section can be summarised in the following diagram:

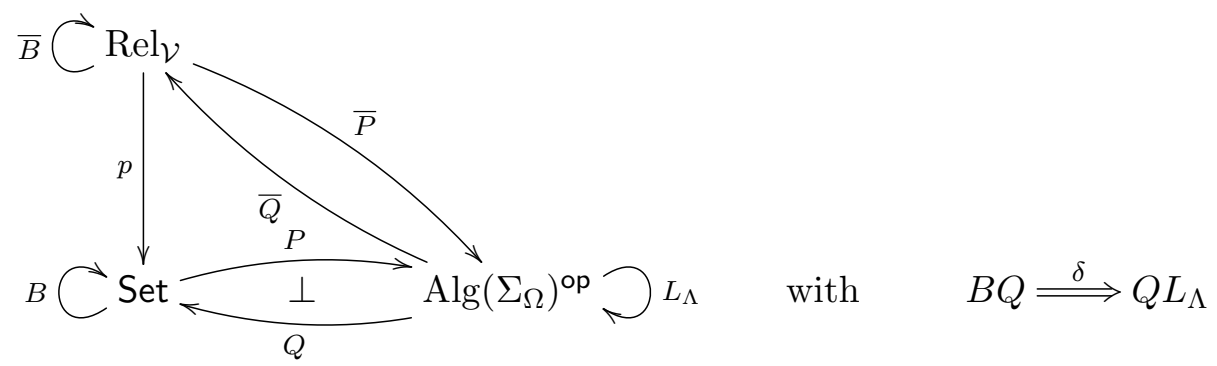

In the next section we will see that adequacy of the logic $\mathcal{L}(\Lambda)$ follows if $\delta$ lifts to $\bar{\delta}: \bar{B} \bar{Q} \Rightarrow \bar{Q} L_{\Lambda}$, while expressiveness is implied by an additional property of $\bar{\delta}$.

\section{Abstract Framework: Adequacy \& Expressiveness}

In this section, we define when a logic is adequate and expressive with respect to a coinductive predicate, and provide sufficient conditions on the logic. Coinductive predicates are expressed abstractly via fibrations and functor lifting, and logic via a contravariant adjunction. Therefore, we make the following assumptions.

Assumption 3.1. Throughout this section, we assume:

(1) (Type of coalgebra) An endofunctor $B: \mathcal{C} \rightarrow \mathcal{C}$ on a category $\mathcal{C}$;

(2) (Coinductive predicate) A CLat ${ }_{\wedge}$-fibration $p: \mathcal{E} \rightarrow \mathcal{C}$ and a lifting $\bar{B}: \mathcal{E} \rightarrow \mathcal{E}$ of $B$;

(3) (Coalgebraic logic) An adjunction $P \dashv Q: \mathcal{C} \leftrightarrows \mathcal{D}^{\mathrm{op}}$, a functor $L: \mathcal{D} \rightarrow \mathcal{D}$ with an initial algebra $\alpha: L(\Phi) \stackrel{\cong}{\rightarrow} \Phi$, and a natural transformation $\delta: B Q \Rightarrow Q L$.

As explained in the introduction, to formulate adequacy and expressiveness, we need one more crucial ingredient: an object that stipulates how collections of formulas should be compared. In the abstract fibrational setting, we assume an object above $Q \Phi$; more systematically, a functor $\bar{Q}$ above $Q$.

Definition 3.2 (Adequacy and Expressiveness). Let $\bar{Q}: \mathcal{D}^{\mathrm{op}} \rightarrow \mathcal{E}$ be a functor such that $p \circ \bar{Q}=Q$. We say the logic $(L, \delta)$ is

- adequate if $\nu\left(\gamma^{*} \circ \bar{B}_{X}\right) \leq t h^{*}(\bar{Q} \Phi)$ for every $B$-coalgebra $(X, \gamma)$;

- expressive if $\nu\left(\gamma^{*} \circ \bar{B}_{X}\right) \geq t h^{*}(\bar{Q} \Phi)$ for every $B$-coalgebra $(X, \gamma)$.

When we need to refer to the functors $\bar{Q}$ or $\bar{B}$ explicitly, we speak about adequacy and expressiveness via $\bar{Q}$ w.r.t. $\bar{B}$. Examples follow in Section 3.2, where classical expressiveness and adequacy w.r.t. bisimilarity is recovered, and Section 4, where other instances are treated. 
Remark 3.3. Definition 3.2 can be generalised to arbitrary poset fibrations, not necessarily assuming complete lattice structure on the fibres, as follows. Adequacy means that for any $B$-coalgebra $(X, \gamma)$, if $R \leq \gamma^{*} \circ \bar{B}_{X}(R)$ then $R \leq t h^{*}(\bar{Q} \Phi)$. Expressiveness means that for any $B$-coalgebra $(X, \gamma)$, we have $t h^{*}(\bar{Q} \Phi) \leq R$ for some $R$ with $R \leq \gamma^{*} \circ \bar{B}_{X}(R)$. In fact, with these definitions, if $(L, \delta)$ is both adequate and expressive then $\gamma^{*} \circ \bar{B}_{X}$ has a greatest fixed point, given by $t h^{*}(\bar{Q} \Phi)$. We prefer to work with CLat $_{\wedge}$-fibrations, since the definition is slightly simpler, and it covers all our examples.

3.1. Sufficient conditions for expressiveness and adequacy. The results below give conditions on $\bar{B}, \bar{Q}$ and primarily the one-step semantics $\delta$ that guarantee expressiveness (Theorem 3.7) and adequacy (Theorem 3.5). For simplicity we fix the functor $\bar{Q}$.

Assumption 3.4. In the remainder of this section we assume a functor $\bar{Q}: \mathcal{D}^{\text {op }} \rightarrow \mathcal{E}$ such that $p \circ \bar{Q}=Q$.

For adequacy, the main idea is to require sufficient conditions to lift $\delta$ to a logic for $\bar{B}$.

Theorem 3.5. Suppose that

(1) $\bar{B} \bar{Q} X \leq \delta_{X}^{*}(\bar{Q} L X)$ for every object $X$ in $\mathcal{D}$, and

(2) the functor $\bar{Q}$ has a left adjoint.

Then $(L, \delta)$ is adequate.

Proof. The first assumption yields a natural transformation $\bar{\delta}: \bar{B} \bar{Q} \Rightarrow \bar{Q} L$, defined on a component $X$ by

$$
\bar{\delta}_{X}=\left(\bar{B} \bar{Q} X \longrightarrow \delta_{X}^{*}(\bar{Q} L X) \stackrel{\tilde{\delta}}{\longrightarrow} \bar{Q} L X\right)
$$

where the left arrow is the inclusion $\bar{B} \bar{Q} X \leq \delta_{X}^{*}(\bar{Q} L X)$, and the right arrow $\widetilde{\delta}$ is the Cartesian morphism to $\bar{Q} L X$ above $\delta_{X}$. It follows that $\bar{\delta}_{X}$ is above $\delta_{X}$. Further, naturality follows from $p$ being faithful (as it is a poset fibration, see Section 2.1) and naturality of $\delta$. Observe that we have thus established $(L, \bar{\delta})$ as a logic for $\bar{B}$-coalgebras, via the adjunction $\bar{P} \dashv \bar{Q}$.

Now let $(X, \gamma)$ be a $B$-coalgebra, and $R=\nu\left(\gamma^{*} \circ \bar{B}_{X}\right)$. Then, in particular, $R \leq$ $\gamma^{*} \circ \bar{B}_{X}(R)$, which is equivalent to a coalgebra $\bar{\gamma}: R \rightarrow \bar{B} R$ above $\gamma: X \rightarrow B X$. The logic $(L, \bar{\delta})$ gives us a theory map $\overline{t h}$ of $(R, \bar{\gamma})$ as the unique map making the following diagram commute.

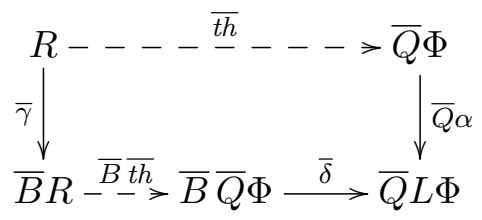

Since $p \circ \bar{Q}=Q$ and $p\left(\bar{\delta}_{\Phi}\right)=\delta_{\Phi}$, it follows that $p(\overline{t h})$ equals the theory map th of $(X, \gamma)$. Hence $R \leq t h^{*}(\bar{Q} \Phi)$ as required.

Expressiveness requires the converse inequality of the one in Theorem 3.5, but only on one component: the carrier $\Phi$ of the initial algebra. Further, the conditions include that $(\bar{B}, B)$ is a fibration map. In particular, for the canonical relation lifting $\operatorname{Rel}(B)$ this means that $B$ should preserve weak pullbacks; this case is explained in more detail in Section 3.2. 
Lemma 3.6. Let $\iota: X \stackrel{\cong}{\rightarrow} Y$ be an isomorphism in $\mathcal{D}$. Then $Q\left(\iota^{-1}\right)^{*}(\bar{Q} Y)=\bar{Q} X$.

Proof. Since $p \circ \bar{Q}=Q$, we have that $\bar{Q}\left(\iota^{-1}\right): \bar{Q} X \rightarrow \bar{Q} Y$ is above $Q\left(\iota^{-1}\right)$, and hence $\bar{Q} X \leq Q\left(\iota^{-1}\right)^{*}(\bar{Q} Y)$ by the latter's universal property. For the converse, consider the following composition, where the left-hand side is the Cartesian morphism:

$$
Q\left(\iota^{-1}\right)^{*}(\bar{Q} Y) \stackrel{\widetilde{Q \iota^{-1}} \bar{Q} Y}{\longrightarrow} \bar{Q} Y \stackrel{\bar{Q} \iota}{\longrightarrow} \bar{Q} X .
$$

This is above the identity on $Q X: p\left(\bar{Q}(\iota) \circ \widetilde{Q \iota^{-1}} \overline{Q Y}\right)=p(\bar{Q}(\iota)) \circ p\left(\widetilde{Q \iota^{-1}} \overline{Q Y}\right)=Q(\iota) \circ Q\left(\iota^{-1}\right)=$ $\operatorname{id}_{Q X}$. Hence we get $Q\left(\iota^{-1}\right)^{*}(\bar{Q} Y) \leq \bar{Q} X$ as needed.

Theorem 3.7. Suppose $(\bar{B}, B)$ is a fibration map. If $\delta_{\Phi}^{*}(\bar{Q} L \Phi) \leq \bar{B} \bar{Q} \Phi$, then $(L, \delta)$ is expressive.

Proof. Let $(X, \gamma)$ be a $B$-coalgebra, with th the associated theory map. We show that $t h^{*}(\bar{Q} \Phi)$ is a post-fixed point of $\gamma^{*} \circ \bar{B}_{X}$ :

$$
\begin{aligned}
t h^{*}(\bar{Q} \Phi) & =\left(Q\left(\alpha^{-1}\right) \circ \delta_{\Phi} \circ B t h \circ \gamma\right)^{*}(\bar{Q} \Phi) \\
& =\gamma^{*} \circ(B t h)^{*} \circ \delta_{\Phi}^{*} \circ Q\left(\alpha^{-1}\right)^{*}(\bar{Q} \Phi) \\
& =\gamma^{*} \circ(B t h)^{*} \circ \delta_{\Phi}^{*}(\bar{Q} L \Phi) \\
& \leq \gamma^{*} \circ(B t h)^{*}(\bar{B} \bar{Q} \Phi) \\
& =\gamma^{*} \circ \bar{B}_{X} \circ t h^{*}(\bar{Q} \Phi)
\end{aligned}
$$

Expressiveness follows since $\nu\left(\gamma^{*} \circ \bar{B}_{X}\right)$ is the greatest post-fixed point.

Note that in the above theorem the reference to the initial algebra $\Phi$ could be avoided by requiring that the inequality in the assumption holds for arbitrary objects in $\mathcal{D}$. We opted for the above formulation reflecting the fact that, whenever one is applying the theorem to concrete instances, it is useful that one is able to focus on the initial $L$-algebra only.

3.2. Adequacy and Expressiveness w.r.t. Behavioural Equivalence. In the setting of coalgebraic modal logic recalled in Section 2.2, Klin [Kli07] proved that

(1) the theory map th of a coalgebra $(X, \gamma)$ factors through coalgebra morphisms from $(X, \gamma)$

(2) if $\delta$ has monic components, then th factors as a coalgebra morphism followed by a mono. The first item can be seen as adequacy w.r.t. behavioural equivalence (i.e., identification by a coalgebra morphism), and the second as expressiveness. ${ }^{1}$

In the current section we revisit this result for Set functors, as a sanity check of Definition 3.2. To obtain the appropriate notion of adequacy and expressiveness, we need to compare collections of formulas for equality. Therefore, the functor $\bar{Q}$ in Definition 3.2 will be instantiated with $\bar{Q} X=\left(Q X, \Delta_{Q X}\right)$ where $\Delta_{Q X}$ denotes the diagonal. Then, for a coalgebra $(X, \gamma), t^{*}(\bar{Q} \Phi)$ is the set of all pairs of states $(x, y)$ such that $\operatorname{th}(x)=\operatorname{th}(y)$. Adequacy then means that for every coalgebra $(X, \gamma)$, behavioural equivalence is contained in $t h^{*}(\bar{Q} \Phi)$, i.e., if $x$ is behaviourally equivalent to $y$ then $t h(x)=t h(y)$. Expressiveness is the converse implication.

\footnotetext{
${ }^{1}$ For weak pullback preserving functors, behavioural equivalence coincides with bisimilarity but for arbitrary set functors the latter can be a strictly smaller relation.
} 
We start with an abstract result, where the functor $\bar{Q}$ assigns the equality relation (diagonal); thus this is specifically about capturing (behavioural) equivalence logically. To state and prove it, let $\Delta$ : Set $\rightarrow$ Rel be the functor given by $\Delta(X)=\Delta_{X}$. This functor has a left adjoint Quot: Rel $\rightarrow$ Set, which maps a relation $R \subseteq X \times X$ to the quotient of $X$ by the least equivalence relation containing $R$ (cf. [HJ98]). This can be generalised to the notion of fibration with quotients, see [Jac99], but we stick to Set here.

Proposition 3.8. Consider the relation fibration $p$ : Rel $\rightarrow$ Set, let $B$ : Set $\rightarrow$ Set be a functor with a lifting $\bar{B}$ : Rel $\rightarrow$ Rel which preserves diagonals, that is, $\bar{B} \circ \Delta=\Delta \circ B$, and such that $(\bar{B}, B)$ is a fibration map.

Let $P \dashv Q:$ Set $\leftrightarrows \mathcal{D}^{\text {op }}$ for some category $\mathcal{D}, L: \mathcal{D} \rightarrow \mathcal{D}$ a functor with an initial algebra and $\delta: B Q \Rightarrow Q L$. Then

(1) $(L, \delta)$ is adequate w.r.t. $\bar{B}$;

(2) if $\delta$ is componentwise injective, then $(L, \delta)$ is expressive w.r.t. $\bar{B}$, via $\bar{Q}=\Delta \circ Q$.

Proof. For adequacy, we use Theorem 3.5. By composition of adjoints, $P \circ$ Quot is a left adjoint to $\Delta \circ Q$. It will be useful to simplify $\bar{B} \circ \Delta \circ Q X$ and $\delta_{X}^{*}(\Delta \circ Q \circ L X)$ :

$$
\begin{aligned}
\bar{B} \circ \Delta \circ Q X & =\Delta_{B Q X}, \\
\delta_{X}^{*}(\Delta \circ Q \circ L X) & =\left(\delta_{X} \times \delta_{X}\right)^{-1}\left(\Delta_{Q L X}\right),
\end{aligned}
$$

using that $\bar{B}$ preserves diagonals in the first equality. The remaining hypothesis of Theorem 3.5 is that $\bar{B} \circ \Delta \circ Q X \leq \delta_{X}^{*}(\Delta \circ Q \circ L X)$ for all $X$, i.e., $\Delta_{B Q X} \subseteq\left(\delta_{X} \times \delta_{X}\right)^{-1}\left(\Delta_{Q L X}\right)$, which is trivial.

For expressiveness, we use Theorem 3.7. By assumption, $(\bar{B}, B)$ is a fibration map. We need to prove that $\delta_{\Phi}^{*}(\Delta \circ Q \circ L \Phi) \leq \bar{B} \circ \Delta \circ Q \Phi$, which amounts to the inclusion

$$
\left(\delta_{\Phi} \times \delta_{\Phi}\right)^{-1}\left(\Delta_{Q L \Phi}\right) \subseteq \Delta_{B Q \Phi}
$$

But this is equivalent to injectivity of $\delta_{\Phi}$.

The canonical lifting $\operatorname{Rel}(B)$ of a Set functor $B$ always preserves diagonals, and if $B$ preserves weak pullbacks, then it is a fibration map. Thus, we obtain expressiveness w.r.t. bisimilarity for weak pullback preserving functors, if $\delta$ has injective components.

In order to be able to cover a larger class of functors, and move to behavioural equivalence, we use the notion of lax extension preserving diagonals.

Definition 3.9 [MV15]. Let $B$ : Set $\rightarrow$ Set be a functor. An operation $\widehat{B}$ associating with each relation $R \subseteq X \times Y$ a relation $\widehat{B} \subseteq B X \times B Y$ is called a lax extension of $B$ preserving diagonals if for all relations $R, S$, all functions $f$ an all sets $X$ we have

(1) $\widehat{B}\left(R^{\bullet}\right)=(\widehat{B} R)^{\bullet}$ where $\left(_{-}\right)^{\bullet}$ denotes the converse relation,

(2) $R \subseteq S$ implies $\widehat{B} R \subseteq \widehat{B} S$ (monotonicity),

(3) $\widehat{B} R ; \widehat{B} S \subseteq \widehat{B}(R ; S)$ (lax preservation of relational composition),

(4) $\widehat{B} \operatorname{Gr}(f)=\operatorname{Gr}(B f)$ and, in particular, $\widehat{B} \Delta_{X}=\Delta_{B X}$ (preservation of diagonals).

Here for a function $f: X \rightarrow Y$ we denote by $\operatorname{Gr}(f)$ its graph relation:

$$
\operatorname{Gr}(f)=\{(x, f(x)) \mid x \in X\} \subseteq X \times Y
$$

The following key fact is an immediate consequence of the results in [MV15]. 
Fact 3.10. Let $B$ be a set functor and let $\widehat{B}$ be a lax extension of $B$ preserving diagonals. Then on any coalgebra $(X, \gamma)$ we have that behavioural equivalence is equal to $\nu\left(\gamma^{*} \circ \widehat{B}_{X}\right)$.

Proof (Sketch). Monotonicity of $\widehat{B}$ implies that $\gamma^{*} \circ \widehat{B}_{X}$ is a monotone operator. The result now follows from $\operatorname{Rel}_{X}$ being a complete lattice and behavioural equivalence being the greatest post-fixed point of $\gamma^{*} \circ \widehat{B}_{X}$. The latter is a consequence of [MV15, Prop. 9].

In particular, for a weak pullback preserving functor $B$, the canonical lifting $\operatorname{Rel}(B)$ is a lax extension preserving diagonals. But the results in [MV15] also show that non-weak pullback preserving set functors have such lax extensions. In fact, any finitary functor for which an expressive logic with "monotone" modalities exist, has a suitable lifting. Examples include the so-called $\left({ }_{-}\right)_{2}^{3}$-functor, the functor $\mathcal{P}_{n}$ that maps a set $X$ to the collection $\mathcal{P}_{n} X$ of subsets of $X$ with less than $n$ elements and the so-called monotone neighbourhood functor (cf. Example 7 in [MV15]). The following proposition establishes that the lax lifting $\widehat{B}$ fits into the fibrational framework of our paper, and that Proposition 3.8 applies.

Proposition 3.11. Let $B$ : Set $\rightarrow$ Set be a functor and let $\widehat{B}$ be a lax lifting of $B$ that preserves diagonals. Then $\widehat{B}: \operatorname{Rel} \rightarrow \operatorname{Rel}$ is a lifting of $B$ along the relation fibration $p:$ Rel $\rightarrow$ Set. In addition to that, $(\widehat{B}, B)$ is a fibration map.

Proof. In order to turn $\widehat{B}$ into a functor Rel $\rightarrow$ Rel we define $\widehat{B}(f):=B f$ - we will verify later in the proof that the functor is well-defined. Now note that for all relations $R \subseteq X \times X$ and functions $f: Y \rightarrow X$ we have

$$
\left.\widehat{B}_{Y}\left(f^{*}(R)\right)\right)=\widehat{B}_{Y}\left((f \times f)^{-1}(R)\right) \stackrel{(*)}{=}(B f \times B f)^{-1}\left(\widehat{B}_{X}(R)\right)=(B f)^{*}\left(\widehat{B}_{X}(R)\right)
$$

where $(*)$ is a well-known property of lax extensions (cf. e.g. Remark 4 in [MV15]) and the other equalities follow from the definition of reindexing. This implies that $(\widehat{B}, B)$ is a fibration map once we establish that $\widehat{B}$ is a lifting of $B$ along $p$ : Rel $\rightarrow$ Set. For the latter we only need to verify that $\widehat{B}$ is a functor on Rel. To avoid confusion, please note that [MV15] uses a different category Rel where the relations are morphisms whereas in our case the relations are objects. In order to see that $\widehat{B}$ is well-defined on Rel-morphisms, consider relations $R \subseteq X \times X, S \subseteq Y \times Y$ and a function $f: R \rightarrow S \in$ Rel. We need to show that $\widehat{B}(f): \widehat{B}(R) \rightarrow \widehat{B}(S)$. As $\widehat{B}(f)=B f$, we need to prove that $B f$ is a Rel-morphism from $\widehat{B}(R)$ to $\widehat{B}(S)$. Consider an arbitrary pair $\left(t_{1}, t_{2}\right) \in \widehat{B}(R)$. We have

$$
\widehat{B}(R) \subseteq \widehat{B}\left((f \times f)^{-1}(S)\right)=(B f \times B f)^{-1}(\widehat{B}(S))
$$

where the inclusion is a consequence of $f$ being a Rel-morphism and monotonicity of $\widehat{B}$, and the equality is an instance of $(*)$. Therefore $\left(t_{1}, t_{2}\right) \in \widehat{B}(R)$ implies $\left(t_{1}, t_{2}\right) \in$ $(B f \times B f)^{-1}[\widehat{B}(S)]$ which is in turn equivalent to $\left(B f\left(t_{1}\right), B f\left(t_{2}\right)\right) \in \widehat{B}(S)$. This shows that $\widehat{B} f: \widehat{B}(R) \rightarrow \widehat{B}(S)$ as required. Functoriality now follows easily from the fact that $\widehat{B} f=B f$ for all functions $f$.

\section{ExAmples}

In this section we instantiate the abstract framework to three concrete examples: a behavioural metric on deterministic automata (Section 4.1), captured by [0,1]-valued tests; a 
unary predicate on transition systems (Section 4.2); and similarity of transition systems, captured by a logic with conjunction and diamond modalities (Section 4.3).

4.1. Shortest distinguishing word distance. We study a simple behavioural distance on deterministic automata: for two states $x, y$ and a fixed constant $c$ with $0<c<1$, the distance is given by $c^{n}$, where $n$ is the length of the smallest word accepted from one state but not the other. Following [BKP18], we refer to this distance as the shortest distinguishing word distance, and, for an automaton with state space $X$, denote it by $d_{s d w}: X \times X \rightarrow[0,1]$.

Formally, fix a finite alphabet $A$, and consider the functor $B$ : Set $\rightarrow$ Set, $B X=2 \times X^{A}$ of deterministic automata. We make use of the fibration $p: \operatorname{Rel}_{[0,1]} \rightarrow$ Set, and define the lifting $\bar{B}: \operatorname{Rel}_{[0,1]} \rightarrow \operatorname{Rel}_{[0,1]}$ by

$$
\bar{B}(X, d)=\left(B X,\left(\left(o_{1}, t_{1}\right),\left(o_{2}, t_{2}\right)\right) \mapsto\left\{\begin{array}{ll}
1 & \text { if } o_{1} \neq o_{2} \\
c \cdot \max _{a \in A}\left\{d\left(t_{1}(a), t_{2}(a)\right)\right\} & \text { otherwise }
\end{array}\right)\right.
$$

The shortest distinguishing word distance $d_{s d w}$ on a deterministic automaton $\gamma: X \rightarrow 2 \times X^{A}$ is the greatest fixed point $\nu\left(\gamma^{*} \circ \bar{B}_{X}\right)$ (recall that in $\operatorname{Rel}_{[0,1]}$ we use the reverse order on $[0,1]$, see Example 2.6).

For an associated logic, we simply use words over $A$ as formulas, and define a satisfaction relation which is weighted in $[0,1]$. Consider the following setting.

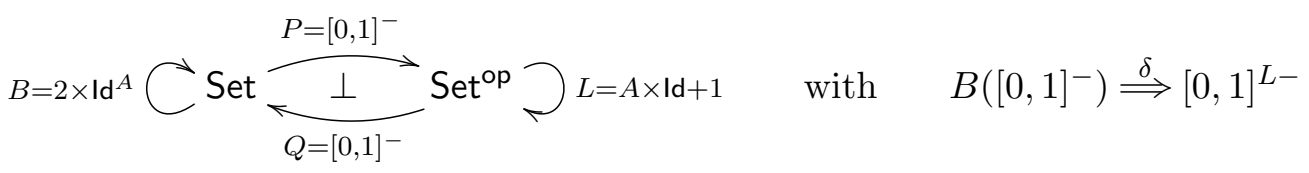

The initial algebra of $L$ is the set of words $A^{*}$. The natural transformation $\delta$ is given by $\delta_{X}: 2 \times\left([0,1]^{X}\right)^{A} \rightarrow[0,1]^{A \times X+1}$,

$$
\delta_{X}(o, t)(u)= \begin{cases}o & \text { if } u=* \in 1 \\ c \cdot t(a)(x) & \text { if } u=(a, x) \in A \times X\end{cases}
$$

which is a quantitative, discounted version of the Boolean-valued logic in Example 2.9. The $\operatorname{logic}(L, \delta)$ defines, for any deterministic automaton $\langle o, t\rangle: X \rightarrow 2 \times X^{A}$, a theory map th: $X \rightarrow[0,1]^{A^{*}}$, given by

$$
\operatorname{th}(x)(\varepsilon)=o(x) \quad \text { and } \quad \operatorname{th}(x)(a w)=c \cdot \operatorname{th}(t(x)(a))(w),
$$

for all $x \in X, a \in A, w \in A^{*}$.

We characterise the shortest distinguishing word distance with the above logic, by instantiating and proving adequacy and expressiveness. Define

$$
\bar{Q}: \operatorname{Set}^{\text {op }} \rightarrow \operatorname{Rel}_{[0,1]}, \quad \bar{Q}(X)=\left([0,1]^{X},\left(\phi_{1}, \phi_{2}\right) \mapsto \sup _{x \in X}\left|\phi_{1}(x)-\phi_{2}(x)\right|\right) .
$$

Technically, this functor is given by mapping a set $X$ to the $X$-fold product of the object $\overline{[0,1]}=([0,1],(r, s) \mapsto|r-s|)$. It follows immediately that $\bar{Q}$ has a left adjoint, mapping $(X, d)$ to $\operatorname{Hom}((X, d), \overline{[0,1]})$, see Equation 2.4. This will be useful for proving adequacy below. 
The functor $\bar{Q}$ yields a 'logical distance' between states $x, y \in X$, given by $t h^{*}(\bar{Q} \Phi)$. We abbreviate it by $d_{l o g}: X \times X \rightarrow[0,1]$. Explicitly, we have

$$
d_{l o g}(x, y)=\sup _{w \in A^{*}}|\operatorname{th}(x)(w)-\operatorname{th}(y)(w)| .
$$

Instantiating Definition 3.2, the logic $(L, \delta)$ is

- adequate if $d_{s d w} \geq d_{l o g}$, and

- expressive if $d_{s d w} \leq d_{l o g}$.

Here $\leq$ is the usual order on $[0,1]$, with 0 the least element (the order in $\operatorname{Re}_{[0,1]}$ is reversed).

To prove adequacy and expressiveness, we use Theorem 3.5 and Theorem 3.7. The functor $\bar{Q}$ has a left adjoint, as explained above. Further, $(\bar{B}, B)$ is a fibration map [BKP18]. We prove the remaining hypotheses of both propositions by showing the equality $\bar{B} \bar{Q} X=$ $\delta_{X}^{*}(\bar{Q} L X)$ for every object $X$ in $\mathcal{D}$. To this end, we compute (suppressing the carrier set $B Q X)$ :

$$
\begin{aligned}
& \delta_{X}^{*}(\bar{Q} L X) \\
= & \left(\left(\left(o_{1}, t_{1}\right),\left(o_{2}, t_{2}\right)\right) \mapsto \sup _{u \in A \times X+1}\left|\delta_{X}\left(o_{1}, t_{1}\right)(u)-\delta_{X}\left(o_{2}, t_{2}\right)(u)\right|\right) \\
= & \left(\left(\left(o_{1}, t_{1}\right),\left(o_{2}, t_{2}\right)\right) \mapsto\left\{\begin{array}{ll}
1 & \text { if } o_{1} \neq o_{2} \\
\left.\sup _{u \in A \times X}\left|\delta_{X}\left(o_{1}, t_{1}\right)(u)-\delta_{X}\left(o_{2}, t_{2}\right)(u)\right|\right) & \text { otherwise }
\end{array}\right)\right. \\
= & \left(\left(\left(o_{1}, t_{1}\right),\left(o_{2}, t_{2}\right)\right) \mapsto\left\{\begin{array}{ll}
1 & \text { if } o_{1} \neq o_{2} \\
\left.\sup _{(a, x) \in A \times X}\left|c \cdot t_{1}(a)(x)-c \cdot t_{2}(a)(x)\right|\right) & \text { otherwise } \\
1 & \text { if } o_{1} \neq o_{2} \\
\left.c \cdot \max _{a \in A} \sup _{x \in X}\left|t_{1}(a)(x)-t_{2}(a)(x)\right|\right) & \text { otherwise }
\end{array}\right)\right. \\
= & \left(( ( o _ { 1 } , t _ { 1 } ) , ( o _ { 2 } , t _ { 2 } ) ) \mapsto \left\{\begin{array}{ll}
B \\
=
\end{array} X\right.\right.
\end{aligned}
$$

Hence, the logic $(L, \delta)$ is adequate and expressive w.r.t. the shortest distinguishing word distance, i.e., $d_{s d w}$ coincides with the logical distance $d_{l o g}$ given in Equation 4.1.

4.2. Divergence of processes. A state of an LTS is said to be diverging if there exists an infinite path of $\tau$-transitions starting at that state. To model this predicate, let $B$ : Set $\rightarrow$ Set, $B X=\left(\mathcal{P}_{\omega} X\right)^{A}$, where $A$ is a set of labels containing the symbol $\tau \in A$. Consider the predicate fibration $p$ : Pred $\rightarrow$ Set, and define the lifting $\bar{B}$ : Pred $\rightarrow$ Pred by

$$
\bar{B}(X, \Gamma)=\left(\left(\mathcal{P}_{\omega} X\right)^{A},\{t \mid \exists x \in \Gamma . x \in t(\tau)\}\right) .
$$

The coinductive predicate defined by $\bar{B}$ on a $B$-coalgebra $(X, \gamma)$ is the set of diverging states:

$$
\nu\left(\gamma^{*} \circ \bar{B}_{X}\right)=(X,\{x \mid x \text { is diverging }\}) .
$$

Now, we want to prove in our framework of adequacy and expressiveness that $x$ is diverging iff for every $n \in \mathbb{N}$ there is a finite path of $\tau$-steps starting in $x$, i.e., $x \models\langle\tau\rangle^{n} \top$ for every $n$. The proof relies on two main observations:

- if $x$ satisfies infinitely many formulas of $\langle\tau\rangle^{n} \top$, then one of its $\tau$-successors does, too;

- if a state $x$ satisfies $\langle\tau\rangle^{n} \top$ for some $n$, then $x$ satisfies $\langle\tau\rangle^{m} \top$ for all $0 \leq m \leq n$.

Combined, one can then give a coinductive proof, showing that if the current state satisfies all formulas of the form $\langle\tau\rangle^{n} T$, then one of its $\tau$-successors also satisfies all these formulas. 
We make this argument precise by casting it into the abstract framework. First, for the logic, we have the following setting:

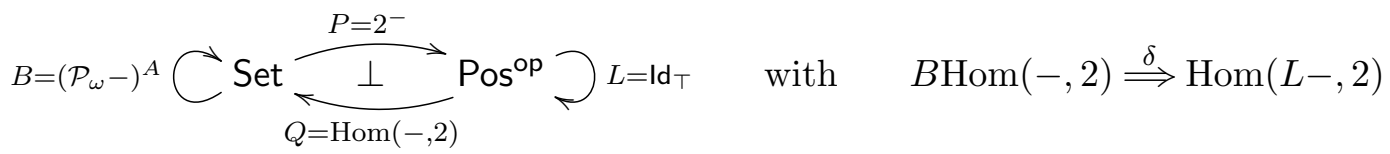

Here Pos is the category of posets and monotone maps, and $2=\{0,1\}$ is the poset given by the order $0 \leq 1$. For a poset $S, \operatorname{Hom}(S, 2)$ is then the set of upwards closed subsets of $S$.

The functor $L S=S_{\top}$ is defined on a poset $S$ by adjoining a new top element $\top$, i.e., the carrier is $S+\{\top\}$ and $\top$ is strictly above all elements of $S$. The initial algebra $\Phi$ of $L$ is the set of natural numbers, representing the formulas of the form $\langle\tau\rangle^{n} T$, linearly ordered, with 0 the top element. The choice of Pos means that the set $\operatorname{Hom}(\Phi, 2)$ used to represent the theory of a state $x \in X$ consists of upwards closed sets (so closed under lower natural numbers in the usual ordering), corresponding to the second observation above concerning the set of formulas satisfied by $x$.

The natural transformation $\delta$ is given by $\delta_{S}:\left(\mathcal{P}_{\omega} \operatorname{Hom}(S, 2)\right)^{A} \rightarrow \operatorname{Hom}\left(S_{\top}, 2\right)$,

$$
\delta_{S}(t)(x)=\left\{\begin{array}{ll}
1 & \text { if } x=\top \\
\bigvee_{\phi \in t(\tau)} \phi(x) & \text { otherwise }
\end{array} .\right.
$$

To show that this is well-defined, suppose $x, y \in S_{\top}$ with $x \leq y$, and suppose $\delta_{S}(t)(x)=1$. If $x=\top$, then $y=\top$, so $\delta_{S}(t)(y)=1$. Otherwise, there is $\phi \in \operatorname{Hom}(S, 2)$ such that $\phi \in t(\tau)$ and $\phi(x)=1$. Since $\phi$ is upwards closed, $\phi(y)=1$ and consequently $\delta_{S}(t)(y)=1$ as needed.

Now, the theory map $t h: X \rightarrow \operatorname{Hom}(\Phi, 2)$ is given by $\operatorname{th}(x)(n)=1$ iff there exists a path of $\tau$-steps of length $n$ from $x$. We define

$$
\bar{Q}: \text { Pos }^{\text {op }} \rightarrow \text { Pred }, \quad \bar{Q}(S)=(\operatorname{Hom}(S, 2),\{\phi \mid \forall x \in S . \phi(x)=1\}) .
$$

Instantiating Definition 3.2, adequacy means that if $x$ is diverging, then $x \models\langle\tau\rangle^{n} \top$ for all $n$; and expressiveness is the converse.

We start with proving adequacy, using Theorem 3.5. The left adjoint $\bar{P}$ is given by $\bar{P}(X, \Gamma)=\left(\operatorname{Hom}((X, \Gamma),(2,\{1\})),\left\{\left(\phi_{1}, \phi_{2}\right) \mid \forall x \in X . \phi_{1}(x) \leq \phi_{2}(x)\right\}\right)$. It remains to prove that $\bar{B} \bar{Q}(S) \leq \delta_{S}^{*}(\bar{Q} L S)$ for all $S$. To this end, we observe $B Q S=\left(\mathcal{P}_{\omega}(\operatorname{Hom}(S, 2))\right)^{A}$ and compute:

$$
\begin{aligned}
\delta_{S}^{*}(\bar{Q} L S) & =\left\{t \mid \delta_{S}(t) \in \bar{Q} L S\right\} \\
& =\left\{t \mid \forall x \in S_{\top} . \delta_{S}(t)(x)=1\right\} \\
& =\left\{t \mid \forall x \in S . \delta_{S}(t)(x)=1\right\} \\
& =\left\{t \mid \forall x \in S . \bigvee_{\phi \in t(\tau)} \phi(x)=1\right\}
\end{aligned}
$$

and $\bar{B} \bar{Q}(S)=\{t \mid(\lambda x .1) \in t(\tau)\}$. The needed inclusion is now trivial.

For expressiveness we have to prove the reverse inclusion with $S=\Phi$, i.e.,

$$
\left\{t \in\left(\mathcal{P}_{\omega}(\operatorname{Hom}(\Phi, 2))\right)^{A} \mid \forall x \in \Phi . \bigvee_{\phi \in t(\tau)} \phi(x)=1\right\} \subseteq\left\{t \in\left(\mathcal{P}_{\omega}(\operatorname{Hom}(\Phi, 2))\right)^{A} \mid(\lambda x .1) \in t(\tau)\right\}
$$

To this end, let $t$ be an element of the left-hand side, and suppose towards a contradiction that for all $\phi$ with $\phi \in t(\tau)$, there is an element $x_{\phi} \in \Phi$ with $\phi\left(x_{\phi}\right)=0$. Choosing an assignment $\phi \mapsto x_{\phi}$ of such elements, we get a finite set $\left\{x_{\phi} \mid \phi \in t(\tau)\right\}$. Let $x_{\phi}$ be the 
smallest element of that set (w.r.t. the order of $\Phi$, i.e., the largest natural number), and let $\psi \in t(\tau)$ be such that $\psi\left(x_{\phi}\right)=1$; such a $\psi$ exists by assumption on $t$. However, since $x_{\phi} \leq x_{\psi}$ and $\psi$ is upwards closed we have $\psi\left(x_{\psi}\right)=1$, which gives a contradiction. Hence, the inclusion holds as required. The lifting $(\bar{B}, B)$ is a fibration map. We thus conclude from Theorem 3.7 that the logic is expressive.

4.3. Simulation of processes. Let $A$ be a set, and define the functor $B$ : Set $\rightarrow$ Set by $B X=\left(\mathcal{P}_{\omega} X\right)^{A}$. Let $\gamma: X \rightarrow\left(\mathcal{P}_{\omega} X\right)^{A}$ be $B$-coalgebra, i.e., a labelled transition system. Denote similarity by $\precsim \subseteq X \times X$, defined more precisely below. Consider the logic with the following syntax:

$$
\varphi, \psi::=\langle a\rangle \varphi|\varphi \wedge \psi| \top
$$

where $a$ ranges over $A$, with the usual interpretation $x \models \varphi$ for states $x \in X$. A classical Hennessy-Milner theorem for similarity is:

$$
x \precsim y \text { iff } \forall \varphi \cdot x \mid=\varphi \rightarrow y \models \varphi .
$$

We show how to formulate and prove this result within our abstract framework.

First, recall from Equation 2.2 in Section 2.1 the appropriate lifting $\bar{B}$ : Rel $\rightarrow$ Rel in the relation fibration $p$ : Rel $\rightarrow$ Set. A simulation on a $B$-coalgebra $(X, \gamma)$ is a relation $R$ such that $R \leq \gamma^{*} \circ \bar{B}_{X}(R)$, and similarity $\precsim$ is the greatest fixed point of $\gamma^{*} \circ \bar{B}_{X}$.

For the logic, to incorporate finite conjunction, we instantiate $\mathcal{D}$ with the category $\mathrm{SL}$ of bounded (meet)-semilattices, i.e., sets equipped with an associative, commutative and idempotent binary operator $\wedge$ and a top element $T$.

To add the modalities $\langle a\rangle$ for each $a \in A$, we proceed as follows. Let $U: \mathrm{SL} \rightarrow$ Set be the forgetful functor. It has a left adjoint $\mathcal{F}$ : Set $\rightarrow$ SL, mapping a set $X$ to the meet-semilattice $\mathcal{P}_{\omega}(X)$ with the top element given by $\emptyset$ and the meet by union. The functor $L: S L \rightarrow S L$ is given by $L X=\mathcal{F}(A \times U X)$; its initial algebra $\Phi$ consists precisely of the language presented in Equation 4.2, quotiented by the semilattice equations ${ }^{2}$. For the adjunction, we use:

$$
B=\left(\mathcal{P}_{\omega}-\right)^{A} \bigodot \operatorname{Set} \underset{Q=\operatorname{Hom}(-, 2)}{\stackrel{P=2^{-}}{\perp}} \mathrm{SL}^{\text {op }} \bigodot_{L=\mathcal{F}(A \times U-)} \text { with } B \operatorname{Hom}(-, 2) \stackrel{\delta}{\Longrightarrow} \operatorname{Hom}(L-, 2)
$$

which is an instance of Equation 2.4. Here $2=\{0,1\}$ is the meet-semilattice given by the order $0 \leq 1$. For a semilattice $S$, the $\operatorname{set} \operatorname{Hom}(S, 2)$ of semilattice morphisms is isomorphic to the set of filters on $S$ : subsets $X \subseteq S$ such that $\top \in X$, and $x, y \in X$ iff $x \wedge y \in X$.

To define the natural transformation $\delta_{S}:\left(\mathcal{P}_{\omega}(\operatorname{Hom}(S, 2))\right)^{A} \rightarrow \operatorname{Hom}(\mathcal{F}(A \times U S), 2)$ on a semilattice $S$, we use that for every map $f: A \times U S \rightarrow 2$ there is a unique semilattice homomorphism $f^{\sharp}: \mathcal{F}(A \times U S) \rightarrow 2$ extending it:

$$
\delta_{S}(t)=\left((a, x) \mapsto \bigvee_{\phi \in t(a)} \phi(x)\right)^{\sharp}=\left(W \mapsto \bigwedge_{(a, x) \in W} \bigvee_{\phi \in t(a)} \phi(x)\right) .
$$

For an LTS $(X, \gamma)$, the associated theory map $t h: X \rightarrow \operatorname{Hom}(\Phi, 2)$ maps a state to the formulas in (4.2) that it accepts, with the usual semantics.

\footnotetext{
${ }^{2}$ To simplify the presentation we do not quotient with monotonicity axioms for the modal operators, i.e., we do not ensure that $\varphi_{1} \leq \varphi_{2}$ implies $\langle a\rangle \varphi_{1} \leq\langle a\rangle \varphi_{2}$.
} 
To recover (4.3), we need to relate logical theories appropriately. Define

$$
\bar{Q}: \mathrm{SL}^{\text {op }} \rightarrow \operatorname{Rel}, \quad \bar{Q} S=\left(\operatorname{Hom}(S, 2),\left\{\left(\phi_{1}, \phi_{2}\right) \mid \forall x \in S . \phi_{1}(x) \leq \phi_{2}(x)\right\}\right) .
$$

Then $t^{*}(\bar{Q} \Phi)=\{(x, y) \mid \forall \varphi \in \Phi . \operatorname{th}(x)(\varphi) \leq t h(y)(\varphi)\}$, i.e., it relates all $(x, y)$ such that the set of formulas satisfied at $x$ is included in the set of formulas satisfied at $y$. Thus, instantiating Definition 3.2, adequacy $\precsim=\nu\left(\gamma^{*} \circ \bar{B}_{X}\right) \leq t h^{*}(\bar{Q} \Phi)$ is the implication from left to right in Equation 4.3, and expressiveness is the converse.

We prove adequacy and expressiveness. The functor $\bar{Q}$ has a left adjoint, given by $\bar{P}(X, R)=\operatorname{Hom}((X, R), \overline{2})$, where $\overline{2}=(2,\{(x, y) \mid x \leq y\})$. This follows by Corollary 2.13 with Remark 2.12, with SL as a full subcategory of the category of all algebras for the corresponding signature.

Given a semilattice $S$, we compute $\delta_{S}^{*}(\bar{Q} L S) \subseteq(B Q S)^{2}=\left(\left(\mathcal{P}_{\omega}(\operatorname{Hom}(S, 2))\right)^{A}\right)^{2}$ :

$$
\begin{aligned}
\delta_{S}^{*}(\bar{Q} L S) & =\delta_{S}^{*}\left(\left\{\left(\phi_{1}, \phi_{2}\right) \mid \forall W \in \mathcal{F}(A \times U S) . \phi_{1}(W) \leq \phi_{2}(W)\right\}\right) \\
& =\left\{\left(t_{1}, t_{2}\right) \mid \forall W \in \mathcal{F}(A \times U S) . \bigwedge_{(a, x) \in W} \bigvee_{\phi \in t_{1}(a)} \phi(x) \leq \bigwedge_{(a, x) \in W} \bigvee_{\phi \in t_{2}(a)} \phi(x)\right\} .
\end{aligned}
$$

Further, $\bar{B} \bar{Q} S=\left\{\left(t_{1}, t_{2}\right) \mid \forall a \in A . \forall \phi_{1} \in t_{1}(a) . \exists \phi_{2} \in t_{2}(a) . \forall x \in S . \phi_{1}(x) \leq \phi_{2}(x)\right\}$. For adequacy, we need to prove $\bar{B} \bar{Q} S \leq \delta_{S}^{*}(\bar{Q} L S)$; but this is trivial, given the above computations. For expressiveness, let $\left(t_{1}, t_{2}\right) \in \delta_{S}^{*}(\bar{Q} L S)$. We need to show that $\left(t_{1}, t_{2}\right) \in$ $\bar{B} \bar{Q} S$. Suppose, towards a contradication, that $\left(t_{1}, t_{2}\right) \notin \bar{B} \bar{Q} S$, i.e., there exist $a \in A$ and $\phi_{1} \in t_{1}(a)$ such that for all $\phi_{2} \in t_{2}(a)$, there is $x \in S$ with $\phi_{1}(x)=1$ and $\phi_{2}(x)=0$. We choose such an element $x_{\phi_{2}}$ for every $\phi_{2} \in t_{2}(a)$. Note that the collection $\left\{x_{\phi_{2}} \mid \phi_{2} \in t_{2}(a)\right\}$ is finite-here we make use of the image-finiteness captured by the functor $B$. Now, consider the conjunction $\psi=\bigwedge_{\phi_{2} \in t_{2}(a)} x_{\phi_{2}} \in S$. Using that $\phi_{1}$ is a homomorphism, we have $\phi_{1}(\psi)=\phi_{1}\left(\bigwedge_{\phi_{2} \in t_{2}(a)} x_{\phi_{2}}\right)=\bigwedge_{\phi_{2} \in t_{2}(a)} \phi_{1}\left(x_{\phi_{2}}\right)=1$, and consequently $\bigvee_{\phi \in t_{1}(a)} \phi(\psi)=1$. We also have $\bigvee_{\phi \in t_{2}(a)} \phi(\psi)=\bigvee_{\phi \in t_{2}(a)} \bigwedge_{\phi_{2} \in t_{2}(a)} \phi\left(x_{\phi_{2}}\right)=0$ since $\phi_{2}\left(x_{\phi_{2}}\right)=0$ for every $\phi_{2} \in t_{2}(a)$. Finally, to arrive at a contradiction, let $W=\{(a, \psi)\}$. Since $\left(t_{1}, t_{2}\right) \in \delta_{S}^{*}(\bar{Q} L S)$ this implies $\bigvee_{\phi \in t_{1}(a)} \phi(\psi) \leq \bigvee_{\phi \in t_{2}(a)} \phi(\psi)$, which is in contradiction with the above. It is easy to check that $(\bar{B}, B)$ is a fibration map (cf. [HJ04]). Hence, we conclude expressiveness from Theorem 3.7.

Remark 4.1. In fact, the expressiveness argument also goes through if we replace SL in the above argument with the category of algebras for the bounded semilattice signature. As pointed out in Sec. 2.3 this can be useful in cases where an axiomatisation of the class of algebras involved is not known. In the concrete case above we opted to work with the well-known category SL instead.

\section{Finite-DePth expressiveness And the KleEne fixed Point theorem}

In Section 3 we formulated expressiveness as an inequality $\nu\left(\gamma^{*} \circ \bar{B}_{X}\right) \geq t h^{*}(\bar{Q} \Phi)$ for all $B$-coalgebras $(X, \gamma)$. The sufficient conditions formulated in Theorem 3.7 ensure that $t h^{*}(\bar{Q} \Phi)$ is a post-fixed point of $\gamma^{*} \circ \bar{B}_{X}$, so that the desired inequality follows. Thereby, that approach relies on the Knaster-Tarski fixed point theorem, constructing the greatest fixed point as the largest post-fixed point.

In the current section we explore a different abstract technique for proving expressiveness, which instead relies on a technique for constructing greatest fixed points which is often 
referred to as Kleene's fixed point theorem. Given a monotone function $\varphi: L \rightarrow L$ on a complete lattice $L$, we construct the chain

$$
\top \geq \varphi(\top) \geq \varphi(\varphi(\top)) \geq \ldots
$$

and take its limit

$$
\bigwedge_{i \in \mathbb{N}} \varphi^{i}(\top)
$$

If $\varphi$ preserves limits of $\omega$-cochains (decreasing sequences indexed by the natural numbers), also referred to as cocontinuity, then this is the greatest fixed point of $\varphi$.

This suggests a different route to expressiveness: we will formulate sufficient conditions to ensure that

$$
\left(\gamma^{*} \circ \bar{B}_{X}\right)^{i}(\top) \geq\left(t h_{i}\right)^{*}\left(\bar{Q} \Phi_{i}\right)
$$

for all $i \in \mathbb{N}$; here $\Phi_{i}$ refers to formulas of modal depth at most $i$, made more precise below using the initial sequence of the functor $L$, and $t h_{i}: X \rightarrow Q \Phi_{i}$ is the associated theory map. The above family of inequalities (indexed by $i$ ) can be thought of as finite-depth expressiveness: it states that the formulas of modal depth at most $i$ are expressive with respect to the $i$-th approximation of the coinductive predicate defined by $\bar{B}$. For instance, that logical equivalence w.r.t. formulas of depth at most $i$ in Hennessy-Milner logic imply $i$-step bisimilarity.

These conditions are sufficient to ensure finite-depth expressiveness - if we then make the additional assumption that $\gamma^{*} \circ \bar{B}_{X}$ is cocontinuous, we obtain proper expressiveness. In the 'Knaster-Tarski' approach to expressiveness of Theorem 3.7, instead, no such assumption is explicitly formulated. So in that approach, cocontinuity is not explicitly assumed. This explains why in some of the examples - for instance similarity of labelled transition systemspart of the argument resembles a proof of cocontinuity.

A remark is in order here. The cocontinuity of $\gamma^{*} \circ \bar{B}_{X}$, which is a functor on a fibre (hence, a monotone map between posets), is of course different from preservation of limits of chains by $\bar{B}$ or $B$. We refer to [HKC18] for a proper study of the relation between these different sequences. The current section is primarily about another the relation between these various sequences and the initial sequence of $L$.

Throughout this section we work again under Assumptions 3.1 and 3.4 concerning our overall categorical setting. We start by recalling the notion of initial and final sequence.

Definition 5.1 (Initial and final sequence). Suppose $\mathcal{D}$ has an initial object 0 . The initial sequence of $L$ is the chain $\left(L^{i} 0\right)_{i \in \mathbb{N}}$, with connecting morphisms $l_{i, j}: L^{i} 0 \rightarrow L^{j} 0$ for all $i \leq j$ defined by $l_{0, j}=!_{L^{j} 0}$ for all $j$ and $l_{i+1, j+1}=L l_{i, j}$. Further, given an algebra $\alpha: L A \rightarrow A$, we inductively define a cocone $\alpha_{i}: L^{i} 0 \rightarrow A$ by $\alpha_{0}=!_{A}$ and $\alpha_{i+1}=\alpha \circ L \alpha_{i}$.

If $\mathcal{C}$ has an initial object 1 , the final sequence of $B$ is defined dually as $\left(B^{i} 1\right)_{i \in \mathbb{N}}$, with the associated connecting morphisms $b_{j, i}: B^{j} 1 \rightarrow B^{i} 1$ for $i \leq j$. Any coalgebra $\gamma: X \rightarrow B X$ defines a cone $\gamma_{i}: X \rightarrow B^{i} 1$ by $\gamma_{0}=!_{X}$ and $\gamma_{i+1}=B \gamma_{i} \circ \gamma$.

If $L$ preserves colimits of $\omega$-chains, of which the initial sequence is an instance, then the colimit $\operatorname{colim}_{i<\omega} L^{i} 0$ carries an initial algebra [Adá74]. Dually if $B$ preserves limits of $\omega$-co-chains, $\lim _{i<\omega} B^{i} 1$ is a final coalgebra. In both cases, the elements of the respective sequences can be thought of as approximations of the initial algebra and final coalgebra, respectively.

For a coalgebra $(X, \gamma)$, we define the cone

$$
\llbracket-\rrbracket_{i}=\left(P \gamma \circ \widehat{\delta}_{X}\right)_{i}: L^{i} 0 \rightarrow P X
$$


as in Definition 5.1 from the algebra $P \gamma \circ \widehat{\delta}_{X}: L P X \rightarrow P X$. Let

$$
t h_{i}: X \rightarrow Q L^{i} 0
$$

be the transpose of $\llbracket-\rrbracket_{i}$. The elements of $L^{i} 0$ are thought of as modal formulas of rank at most $i$, and $t h_{i}$ is the theory map of a coalgebra restricted to those formulas. It is easy to show that the $t_{i}$ maps satisfy the following properties:

Lemma 5.2. For all $i$, the two triangles in the following diagram commute.

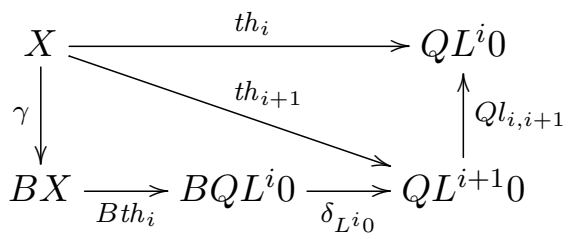

Furthermore we define a sequence $\left(\delta_{i}: B^{i} 1 \rightarrow Q L^{i} 0\right)_{i \in \mathbb{N}}$, which iterates $\delta$ on the final sequence of $B$, as follows:

$$
\delta_{0}=\mathrm{id}: 1 \rightarrow Q 0 \quad \delta_{i+1}=\left(B B^{i} 1 \stackrel{B \delta_{i}}{\longrightarrow} B Q L^{i} 0 \stackrel{\delta_{L^{i} 0}}{\longrightarrow} Q L^{i+1} 0\right) .
$$

We use here that $Q 0=1$ is a final object, as 0 is initial and $Q$ a right adjoint. This enables us to relate $t h_{i}$ and $\gamma_{i}$.

Lemma 5.3. Let $(X, \gamma)$ be a coalgebra. For all $i$, th ${ }_{i}=\delta_{i} \circ \gamma_{i}$.

Proof. By induction on $i$. The base case is trivial: $t h_{0}=!_{X}=\delta_{0} \circ \gamma_{0}$. Suppose it holds for some $i$. Then: $t h_{i+1}=\delta_{L^{i} 0} \circ B t h_{i} \circ \gamma=\delta_{L^{i} 0} \circ B \delta_{i} \circ B \gamma_{i} \circ \gamma=\delta_{i+1} \circ \gamma_{i+1}$, where the first equality holds by Lemma 5.2.

The following lemma shows that, for a coalgebra $(X, \gamma)$, the elements of the final sequence of $\gamma^{*} \circ \bar{B}_{X}$ (in the fibre $\mathcal{E}_{X}$ ) can be retrieved from the final sequence of $\bar{B}$ (in the total category $\mathcal{E}$ ) by reindexing along the maps $\gamma_{i}: X \rightarrow B^{i} 1$.

Lemma 5.4. Suppose $(B, \bar{B})$ is a fibration map, and let $(X, \gamma)$ be a B-coalgebra. Then for all $i,\left(\gamma_{i}\right)^{*}\left(\bar{B}^{i} 1\right)=\left(\gamma^{*} \circ \bar{B}_{X}\right)^{i}(\top)$.

Proof. By induction on $i$. The base case is easy, since reindexing in $\mathrm{CLat}_{\wedge}-$-fibrations preserves top elements. For the inductive case, suppose it holds for some $i$. We compute:

$$
\begin{aligned}
\left(\gamma_{i+1}\right)^{*}\left(\bar{B}^{i+1} 1\right) & =\left(B \gamma_{i} \circ \gamma\right)^{*}\left(\bar{B} \bar{B}^{i} 1\right) \\
& =\gamma^{*} \circ\left(B \gamma_{i}\right)^{*}\left(\bar{B} \bar{B}^{i} 1\right) \\
& =\gamma^{*} \circ \bar{B}_{X} \circ\left(\gamma_{i}\right)^{*}\left(\bar{B}^{i} 1\right) \\
& =\gamma^{*} \circ \bar{B}_{X} \circ\left(\gamma \circ \bar{B}_{X}\right)^{i}(\top) \\
& =\left(\gamma \circ \bar{B}_{X}\right)^{i+1}(\top) .
\end{aligned}
$$

The following result now establishes a sufficient condition on $\delta$ for finite-depth expressiveness, formulated in terms of the final sequence of $\bar{B}$ and initial sequence of $L$.

Proposition 5.5 (Finite-depth expressiveness). Suppose $(\bar{B}, B)$ is a fibration map. Then for all $i$ : if $\delta_{i}^{*}\left(\bar{Q} L^{i} 0\right) \leq \bar{B}^{i} 1$ then for any $B$-coalgebra $(X, \gamma)$, we have $\left(t_{i}\right)^{*}\left(\bar{Q} L^{i} 0\right) \leq$ $\left(\gamma^{*} \circ \bar{B}_{X}\right)^{i}(\top)$. 
Proof. If $\delta_{i}^{*}\left(\bar{Q} L^{i} 0\right) \leq \bar{B}^{i} 1$, then:

$$
\begin{aligned}
\left(t h_{i}\right)^{*}\left(\bar{Q} L^{i} 0\right) & =\left(\gamma_{i}\right)^{*} \circ\left(\delta_{i}\right)^{*}\left(\bar{Q} L^{i} 0\right) \\
& \leq\left(\gamma_{i}\right)^{*}\left(\bar{B}^{i} 1\right) \\
& =\left(\gamma \circ \bar{B}_{X}\right)^{i}(\top) .
\end{aligned}
$$

A natural way to move from the above result on finite-depth expressiveness to full expressiveness is to assume that the functors $\bar{B}_{X}$ on the fibre preserve limits of $\omega$-chains. Note that these are functors on fibres (that is, monotone functions), and there is no assumption on $B$ or $\bar{B}$ preserving anything.

Theorem 5.6 (Expressiveness via Kleene fixed point theorem). Suppose $(\bar{B}, B)$ is a fibration map, and for all $i: \delta_{i}^{*}\left(\bar{Q} L^{i} 0\right) \leq \bar{B} \bar{Q} 0$. Then for any $B$-coalgebra $(X, \gamma)$ and for all $i$ : $t h^{*}(\bar{Q} \Phi) \leq\left(\gamma^{*} \circ \bar{B}_{X}\right)^{i}(\top)$. In particular, if $\bar{B}_{X}$ preserves limits of $\omega$-cochains, then the logic $(L, \delta)$ is expressive.

Proof. Let $(X, \gamma)$ be a coalgebra; we need to prove that $t h^{*}(\bar{Q} \Phi) \leq \nu\left(\gamma^{*} \circ \bar{B}_{X}\right)$. For the initial algebra $(\Phi, \alpha)$, we have the sequence $\alpha_{i}: L^{i} 0 \rightarrow \Phi$ (Definition 5.1) and since $\llbracket-\rrbracket: \Phi \rightarrow P X$ is an algebra morphism, it follows that $\llbracket-\rrbracket \circ \alpha_{i}=\llbracket-\rrbracket_{i}$ for all $i$. Hence, $t h_{i}=Q \alpha_{i} \circ t h: X \rightarrow Q L^{i} 0$. Now, since there is the composite morphism

$$
t h^{*}(\bar{Q} \Phi) \stackrel{\widetilde{t h}_{\bar{Q} \Phi}}{\longrightarrow} \bar{Q} \Phi \stackrel{\bar{Q} \alpha_{i}}{\longrightarrow} \bar{Q} L^{i} 0
$$

above $Q \alpha_{i} \circ t h=t h_{i}$, we have $t h^{*}(\bar{Q} \Phi) \leq t h_{i}^{*}\left(\bar{Q} L^{i} 0\right)$. Combined with Proposition 5.5, we get

$$
t h^{*}(\bar{Q} \Phi) \leq\left(\gamma^{*} \circ \bar{B}_{X}\right)^{i}(\top)
$$

for all $i$.

Finally, if $\bar{B}_{X}$ preserves limits of $\omega$-cochains, then so does $\gamma^{*} \circ \bar{B}_{X}$ (reindexing $\gamma^{*}$ preserves all meets, by the definition of CLat $\wedge_{\wedge}$-fibration). Hence $\bigwedge_{i \in \mathbb{N}}\left(\gamma^{*} \circ \bar{B}_{X}\right)^{i}(\top)=\nu\left(\gamma^{*} \circ \bar{B}_{X}\right)$. And thus $t h^{*}(\bar{Q} \Phi) \leq \nu\left(\gamma^{*} \circ \bar{B}_{X}\right)$.

Example 5.7. We show finite-depth expressiveness (Prop. 5.5) via the above approach for the example of similarity of labelled transition systems (Section 4.3). The relevant endofunctors $B=\mathcal{P}_{\omega}(-)^{A}, \bar{B}, L$, adjunctions $P \dashv Q$ and $\bar{P} \dashv \bar{Q}$, and $\delta$ are all as defined there. Contrary to the treatment in Section 4.3, with the current approach it matters quite a bit whether $A$ is finite or not. ${ }^{3}$ For the moment, we will assume that $A$ is finite, which significantly simplifies the matter. In Example 5.8 below we discuss the infinite case.

The final sequence of $\bar{B}$ is concretely described as a sequence of relations on $B^{i} 1$, by just instantiating $\bar{B}$ for the inductive case:

$$
\bar{B} \bar{B}^{i} 1=\left\{\left(t_{1}, t_{2}\right) \mid \forall a \in A . \forall x \in t_{1}(a) . \exists y \in t_{2}(a) .(x, y) \in \bar{B}^{i} 1\right\} .
$$

and $\bar{B}^{0} 1=\{(*, *)\} \subseteq 1 \times 1$. Thus, $\left(t_{1}, t_{2}\right) \in \bar{B}^{i} 1$ iff $t_{1}$ is " $i$-step simulated" by $t_{2}$, where both $t_{1}$ and $t_{2}$ are viewed as trees of height at most $i$.

The initial sequence of $L: S L \rightarrow S L$ is characterised, once again by spelling out the definition, by $L^{0} 0=0=\{\top\}$ (the one-element semilattice, which is the initial object in $\mathrm{SL}$ ) and $L L^{i} 0=\mathcal{P}_{\omega}\left(A \times L^{i} 0\right)$ (the free semilattice, see Section 4.3). Concretely, elements of

\footnotetext{
${ }^{3}$ This was pointed out to us by Yuichi Komorida.
} 
$L^{i} 0$ can be identified with formulas of depth at most $i$ in the logic of Section 4.3 (diamond modalities and conjunction), quotiented by the semilattice equations. By the assumption that $A$ is finite, each set $L^{i} 0$ is finite.

We continue to prove the main hypothesis of Proposition 5.5 and Theorem 5.6: that

$$
\delta_{i}^{*}\left(\bar{Q} L^{i} 0\right) \leq \bar{B}^{i} 1
$$

for all $i$. Before doing so, we spell out $\delta_{i}^{*}\left(\bar{Q} L^{i} 0\right)$ in some more detail. First, we characterise $\delta_{i+1}: B B^{i} 1 \rightarrow Q L L^{i} 0:$

$$
\begin{aligned}
\delta_{i+1}(t)(W) & =\delta_{L^{i} 0}\left(B \delta_{i}(t)\right)(W) \\
& =\bigwedge_{(a, \psi) \in W} \bigvee_{\phi \in\left(B \delta_{i}(t)\right)(a)} \phi(\psi) \\
& =\bigwedge_{(a, \psi) \in W} \bigvee_{x \in t(a)} \delta_{i}(x)(\psi)
\end{aligned}
$$

The map $\delta_{i}$ assigns to an element $t \in B^{i} 1$ the formulas of modal depth at most $i$ that hold for $t$, viewed as a tree.

We now prove (5.1) by induction on $i$. The base case is trivial. For the inductive case, assume (5.1) holds for some $i$. We have to prove that, for all $\left(t_{1}, t_{2}\right) \in \delta_{i+1}^{*}\left(\bar{Q} L^{i+1} 0\right), a \in A$ and $x \in t_{1}(a)$, there exists $y \in t_{2}(a)$ such that $(x, y) \in B^{i} 1$.

Spelling out the definition of $\delta_{i}^{*}\left(\bar{Q} L^{i} 0\right)$ yields

$$
\delta_{i}^{*}\left(\bar{Q} L^{i} 0\right)=\left\{\left(t_{1}, t_{2}\right) \in \bar{B}^{i} 1 \mid \forall \phi \in L^{i} 0 . \delta_{i}\left(t_{1}\right)(\phi) \leq \delta_{i}\left(t_{2}\right)(\phi)\right\} .
$$

For the $i+1$ case we can expand this further using the above equation for $\delta_{i+1}$ :

$$
\delta_{i+1}^{*}\left(\bar{Q} L^{i+1} 0\right)=\left\{\left(t_{1}, t_{2}\right) \mid \forall W \in L L^{i} 0 . \bigwedge_{(a, \psi) \in W} \bigvee_{x \in t_{1}(a)} \delta_{i}(x)(\psi) \leq \bigwedge_{(a, \psi) \in W} \bigvee_{x \in t_{2}(a)} \delta_{i}(x)(\psi)\right\}
$$

Now, let $\left(t_{1}, t_{2}\right) \in \delta_{i+1}^{*}\left(\bar{Q} L^{i+1} 0\right), a \in A$ and $x \in t_{1}(a)$. Let $\Psi=\left\{\psi \in L^{i} 0 \mid \delta_{i}(x)(\psi)=1\right\}$. Note that this is indeed a finite set, as $L^{i} 0$ is, so we will be able to consider its conjunction. Since $\delta_{i}(x)$ is a filter, we get $\delta_{i}(x)\left(\bigwedge_{\psi \in \Psi} \psi\right)=\bigwedge_{\psi \in \Psi} \delta_{i}(x)(\psi)=1$, where the second equality holds by definition of $\Psi$. Since $\left(t_{1}, t_{2}\right) \in \delta_{i+1}^{*}\left(\bar{Q} L^{i+1} 0\right)$, taking $W=\{(a, \bigwedge \Psi)\}$ (this represents $\left.\langle a\rangle \bigwedge_{\delta_{i}(x)(\psi)=1} \psi\right)$ we get that there exists $y \in t_{2}(a)$ such that $\delta_{i}(y)\left(\bigwedge_{\psi \in \Psi} \psi\right)=1$. Hence, since $\delta_{i}(y)$ is a filter, we get $\delta_{i}(y)(\psi)=1$ for all $\psi \in \Psi$. Thus $(x, y) \in \delta_{i}^{*}\left(\bar{Q} L^{i} 0\right)$, and by the induction hypothesis we obtain $(x, y) \in \bar{B}^{i} 1$ as needed.

The above proof relies on the assumption that the set of labels $A$ is finite. In the following example we show a way to adapt the proof to the case where this assumption is dropped.

Example 5.8. If $A$ is not assumed to be infinite, the above proof does not work, as the meet $\bigwedge \Psi$ may be infinite, which is not defined as we are working with semilattices. To remedy this, with $\Psi$ defined as above, let $\Psi_{0} \subseteq \Psi_{1} \subseteq \Psi_{2} \subseteq \ldots$ be an increasing sequence of finite subsets of $\Psi$ such that $\bigcup_{i \in \mathbb{N}} \Psi_{i}=\Psi$. First note that, for each $i$, we have $\delta_{i}(x)\left(\bigwedge_{\psi \in \Psi_{i}} \psi\right)=1$ using again the filter property. Now, consider

$$
W_{i}=\left\{\left(a, \bigwedge \Psi_{i}\right)\right\}
$$


for $i \in \mathbb{N}$. Following the earlier reasoning, we get for each $i$ an element $y \in t_{2}(a)$ such that $\delta_{i}(y)\left(\bigwedge_{\psi \in \Psi_{i}} \psi\right)=1$. In fact, since $t_{2}(a)$ is finite, there exists an $y$ such that $\delta_{i}(y)\left(\bigwedge_{\psi \in \Psi_{i}} \psi\right)=$ 1 for infinitely many $i$ (and as a consequence for all $i$ ). Since $\delta_{i}(y)$ is a filter this means $\bigwedge_{\psi \in \Psi_{i}} \delta_{i}(y)(\psi)=1$ for infinitely many $i$. Finally, since every $\psi \in \Psi$ is contained in some of these sets $\Psi_{i}$ we obtain the desired result that $\delta(y)(\psi)=1$ for all $\psi \in \Psi$. The proof then concludes as above.

\section{FUTURE WORK}

We proposed suitable notions of expressiveness and adequacy, connecting coinductive predicates in a fibration to coalgebraic modal logic in a contravariant adjunction. Further, we gave sufficient conditions on the one-step semantics that guarantee expressiveness and adequacy, and showed how to put these methods to work in concrete examples.

There are several avenues for future work. First, an intriguing question is whether the characterisation of behavioural metrics in [KM18, WSPK18] can be covered in the setting of this paper, as well as logics for other distances such as the (abstract, coalgebraic) Wasserstein distance. Those behavioural metrics are already framed in a fibrational setting [BKP18, SKDH18, BBKK18, $\left.\mathrm{KKH}^{+} 19\right]$. While all our examples are for coalgebras in Set, the fibrational framework allows different base categories, which might be useful to treat, e.g., behavioural metrics for continuous probabilistic systems [vBW05].

A further natural question is whether we can automatically derive logics for a given predicate. As mentioned in the introduction, there are various tools to find expressive logics for behavioural equivalence. But extending this to the current general setting is non-trivial. Conversely, given a logic, one would like to associate a lifting to it, perhaps based on techniques related to $\Lambda$-bisimulations [GS13, BH17, Enq13].

\section{REFERENCES}

[Adá74] Jiří Adámek. Free algebras and automata realizations in the language of categories. Commentationes Mathematicae Universitatis Carolinae, 15(4):589-602, 1974.

[BBKK18] Paolo Baldan, Filippo Bonchi, Henning Kerstan, and Barbara König. Coalgebraic behavioral metrics. Logical Methods in Computer Science, 14(3), 2018.

[BD16] Marta Bílková and Matej Dostál. Expressivity of many-valued modal logics, coalgebraically. In Jouko A. Väänänen, Åsa Hirvonen, and Ruy J. G. B. de Queiroz, editors, WoLLIC, volume 9803 of LNCS, pages 109-124. Springer, 2016.

[BH17] Zeinab Bakhtiari and Helle Hvid Hansen. Bisimulation for weakly expressive coalgebraic modal logics. In Filippo Bonchi and Barbara König, editors, CALCO, volume 72 of LIPIcs, pages 4:1-4:16. Schloss Dagstuhl - Leibniz-Zentrum fuer Informatik, 2017.

[BK05a] Marcello M. Bonsangue and Alexander Kurz. Duality for logics of transition systems. In Vladimiro Sassone, editor, FoSSaCS, volume 3441 of LNCS, pages 455-469. Springer, 2005.

[BK05b] Marcello M. Bonsangue and Alexander Kurz. Duality for logics of transition systems. In FoSSaCS'05, 2005.

[BKP18] Filippo Bonchi, Barbara König, and Daniela Petrisan. Up-to techniques for behavioural metrics via fibrations. In Schewe and Zhang [SZ18], pages 17:1-17:17.

[BPPR17] Filippo Bonchi, Daniela Petrisan, Damien Pous, and Jurriaan Rot. A general account of coinduction up-to. Acta Inf., 54(2):127-190, 2017.

[CFKP19] Florence Clerc, Nathanaël Fijalkow, Bartek Klin, and Prakash Panangaden. Expressiveness of probabilistic modal logics: A gradual approach. Inf. Comput., 267:145-163, 2019.

[DEP02] Josee Desharnais, Abbas Edalat, and Prakash Panangaden. Bisimulation for labelled markov processes. Inf. Comput., 179(2):163-193, 2002. 
[DGJP99] Josée Desharnais, Vineet Gupta, Radha Jagadeesan, and Prakash Panangaden. Metrics for labeled markov systems. In CONCUR, volume 1664 of Lecture Notes in Computer Science, pages 258-273. Springer, 1999.

[DMS19] Ulrich Dorsch, Stefan Milius, and Lutz Schröder. Graded Monads and Graded Logics for the Linear Time - Branching Time Spectrum. In CONCUR, 2019.

[Enq13] Sebastian Enqvist. Homomorphisms of coalgebras from predicate liftings. In Heckel and Milius [HM13], pages 126-140.

[GS13] Daniel Gorín and Lutz Schröder. Simulations and bisimulations for coalgebraic modal logics. In Heckel and Milius [HM13], pages 253-266.

[Her74] Horst Herrlich. Topological functors. General Topology and its Applications, 4(2):125 - 142, 1974.

[HJ98] Claudio Hermida and Bart Jacobs. Structural induction and coinduction in a fibrational setting. Information and Computation, 145(2):107-152, 1998.

[HJ04] Jesse Hughes and Bart Jacobs. Simulations in coalgebra. Theor. Comput. Sci., 327(1-2):71-108, 2004.

[HKC18] Ichiro Hasuo, Toshiki Kataoka, and Kenta Cho. Coinductive predicates and final sequences in a fibration. Mathematical Structures in Computer Science, 28(4):562-611, 2018.

[HM85] Matthew Hennessy and Robin Milner. Algebraic laws for nondeterminism and concurrency. $J$. ACM, 32(1):137-161, 1985.

[HM13] Reiko Heckel and Stefan Milius, editors. CALCO, volume 8089 of LNCS. Springer, 2013.

[Jac99] Bart Jacobs. Categorical Logic and Type Theory. Elsevier, 1999.

[Jac16] Bart Jacobs. Introduction to Coalgebra: Towards Mathematics of States and Observation, volume 59 of Cambridge Tracts in Theoretical Computer Science. Cambridge University Press, 2016.

[JS09] Bart Jacobs and Ana Sokolova. Exemplaric Expressivity of Modal Logics. Journal of Logic and Computation, 20(5):1041-1068, 2009.

$\left[\mathrm{KKH}^{+} 19\right]$ Yuichi Komorida, Shin-ya Katsumata, Nick Hu, Bartek Klin, and Ichiro Hasuo. Codensity games for bisimilarity. In LICS, pages 1-13. IEEE, 2019.

[KKP04] Clemens Kupke, Alexander Kurz, and Dirk Pattinson. Algebraic semantics for coalgebraic logics. Electr. Notes Theor. Comput. Sci., 106:219-241, 2004.

[KKV12] Krzysztof Kapulkin, Alexander Kurz, and Jiri Velebil. Expressiveness of positive coalgebraic logic. In Thomas Bolander, Torben Braüner, Silvio Ghilardi, and Lawrence S. Moss, editors, AiML, pages 368-385. College Publications, 2012.

[Kli07] Bartek Klin. Coalgebraic modal logic beyond sets. Electr. Notes Theor. Comput. Sci., 173:177-201, 2007.

[KM18] Barbara König and Christina Mika-Michalski. (metric) bisimulation games and real-valued modal logics for coalgebras. In Schewe and Zhang [SZ18], pages 37:1-37:17.

[KP11] Clemens Kupke and Dirk Pattinson. Coalgebraic semantics of modal logics: An overview. Theor. Comput. Sci., 412(38):5070-5094, 2011.

[KR20] Clemens Kupke and Jurriaan Rot. Expressive logics for coinductive predicates. In CSL, volume 152 of LIPIcs, pages 26:1-26:18. Schloss Dagstuhl - Leibniz-Zentrum für Informatik, 2020.

[LS91] Kim Guldstrand Larsen and Arne Skou. Bisimulation through probabilistic testing. Inf. Comput., 94(1):1-28, 1991.

[MV15] Johannes Marti and Yde Venema. Lax extensions of coalgebra functors and their logic. Journal of Computer and System Sciences, 81(5):880 - 900, 2015. CMCS 2012 (Selected Papers).

[Pat04] Dirk Pattinson. Expressive logics for coalgebras via terminal sequence induction. Notre Dame Journal of Formal Logic, 45(1):19-33, 2004.

[PMW06] Dusko Pavlovic, Michael W. Mislove, and James Worrell. Testing semantics: Connecting processes and process logics. In Michael Johnson and Varmo Vene, editors, AMAST, volume 4019 of LNCS, pages 308-322. Springer, 2006.

[PT91] Hans-E. Porst and Walter Tholen. Concrete Dualities. In H. Herrlich and H. E. Porst, editors, Categories at Work, pages 111-136. Heldermann Verlag, 1991.

[Rut00] Jan J. M. M. Rutten. Universal coalgebra: a theory of systems. Theor. Comput. Sci., 249(1):3-80, 2000 .

[Sch08] Lutz Schröder. Expressivity of coalgebraic modal logic: The limits and beyond. Theor. Comput. Sci., 390(2-3):230-247, 2008. 
[SKDH18] David Sprunger, Shin-ya Katsumata, Jérémy Dubut, and Ichiro Hasuo. Fibrational bisimulations and quantitative reasoning. In Corina Cîrstea, editor, CMCS, volume 11202 of LNCS, pages 190-213. Springer, 2018.

[SZ18] Sven Schewe and Lijun Zhang, editors. CONCUR, volume 118 of LIPIcs. Schloss Dagstuhl Leibniz-Zentrum fuer Informatik, 2018.

[vBW05] Franck van Breugel and James Worrell. A behavioural pseudometric for probabilistic transition systems. Theor. Comput. Sci., 331(1):115-142, 2005.

[vG90] Rob J. van Glabbeek. The linear time-branching time spectrum (extended abstract). In Jos C. M. Baeten and Jan Willem Klop, editors, CONCUR, volume 458 of LNCS, pages 278-297. Springer, 1990.

[Wil13] Toby Wilkinson. Enriched coalgebraic modal logic. PhD thesis, University of Southampton, May 2013.

[WSPK18] Paul Wild, Lutz Schröder, Dirk Pattinson, and Barbara König. A van Benthem theorem for fuzzy modal logic. In Anuj Dawar and Erich Grädel, editors, LICS, pages 909-918. ACM, 2018. 